Hydroécol. Appl. (1994) Tome 6 Vol 1-2, pp. 329-368

\title{
Etude du régime thermique du lac de Pareloup avec le modèle EOLE
}

\section{Study of the thermal dynamics of Pareloup lake using the EOLE model}

\author{
Marie-José Salençon(*) \\ (1) Electricité de France, Direction des Etudes et Recherches. Département Environnement. \\ 6 Quai Watier, F-78401 Chatou Cedex, France.
}

Résumé. - EOLE est un modèle hydrodynamique et thermique (modèle unidimensionnel vertical) qui simule l'évolution de la stratification thermique saisonnière d'un réservoir. C'est un modèle à bilan d'énergie, basé sur la représentation des mécanismes physiques, qui prend en compte la bathymétrie de la retenue, les échanges d'énergie à l'interface air-eau ainsi que les entrées et sorties d'eau (rivières, pompages, turbinages, débits réservés etc.).

EOLE a été développé sur le lac de Pareloup, retenue hydroélectrique au long temps de séjour ( $1 \mathrm{an}$ ), en simulant très correctement son régime thermique pendant 12 années consécutives. Le bon accord obtenu entre les profils thermiques calculés et mesurés montre que le modèle a permis de très bien reproduire la stratification thermique de cette retenue, la dynamique verticale des masses d'eau, le régime thermique des eaux restituées à l'aval, ainsi que les fluctuations interannuelles sur de longues périodes (plusieurs cycles annuels).

Le modèle a été appliqué avec les mêmes coefficients dans d'autres cas d'étude en donnant des résultats satisfaisants, ce qui montre que les phénomènes prépondérants de l'hydrodynamique de ces réservoirs sont bien pris en compte. En effet, basé sur la représentation des processus, le modèle n'a pas besoin de calage pourvu que les données d'entrées soient bien représentatives du site étudié, ce qui souligne l'importance de la qualité de ces données d'entrée.

La précision des résultats obtenus alliée à la relative simplicité de la représentation nous a permis d'utiliser ce modèle comme structure de base d'un modèle d'écosystème.

Mots-clés. - réservoir, modèle hydrodynamique et thermique, unidimensionnel, vertical, intégral, bilan d'énergie, couche de mélange. 
Abstract. - EOLE is a hydrodynamic and thermal model (one-dimensional vertical model) which simulates the evolution in seasonal thermal stratification of a reservoir. It is an energy budget model based on representation of physical mechanisms, which takes into account the bathymetry of the reservoir, surface energy exchanges, and throughflow in the reservoir (rivers, pumping, turbining, residual stream flow, etc.).

EOLE was developed on Pareloup Lake, a hydroelectric reservoir with a long residence time (1 year), and proved to simulate the thermal dynamics very correctly over a period of 12 consecutive years. The good agreement between the calculated and measured thermal profiles indicates that the model enables very good reproduction of the thermal stratification in the reservoir, the vertical dynamics of water masses, the thermal regime of water restituted downstream, and year-to-year fluctuations over extended periods (several one-year cycles).

The model was applied using the same coefficients in other studies with good results, demonstrating that the predominant hydrodynamic phenomena in this type of reservoir are well taken into account. In fact, given its representation of the processes, the model needs no calibration providing the input data are sufficiently representative of the site under study, which underscores the importance of the quality of input data.

The precision of the results obtained and the relative simplicity of the EOLE representation have enabled us to use the model as a basic structure for modeling of an ecosystem.

Key-words. - reservoir, hydrodynamic and thermal model, one-dimensional, vertical, integral, energy budget, well-mixed layer.

\section{INTRODUCTION}

L'objectif des modèles thermiques de lacs et réservoirs est de bien représenter l'évolution annuelle de la stratification thermique et ses conséquences sur l'écosystème.

Un certain nombre de modèles a été construit, dont la base est un modèle thermique unidimensionnel (Markofsky et Harleman, 1971; Stefan et Ford, 1975; Hurley Octavio et al., 1977; Imberger et al., 1978; Enderlé, 1980; Ford et Stefan, 1980; Enderlé, 1982; Harleman, 1982; Spigel et al., 1986; Tassin, 1986; Vinçon-Leite et al., 1988; Salençon, 1994).
Actuellement, c'est toujours le modèle unidimensionnel vertical qui est le plus couramment utilisé car il représente bien la structure thermique verticale d'une masse d'eau, qui est le traceur de ses mouvements internes, en réaction aux conditions extérieures : hydrauliques et météorologiques. De plus, il constitue un outil éprouvé, suffisamment fin pour bien reproduire les phénomènes prépondérants mais également suffisamment simple pour pouvoir être couplé à un modèle biologique et ensuite utilisé comme outil de simulation de scénarios sans atteindre des coûts d'exploitation prohibitifs.

L'hypothèse d'unidimensionnalité verticale est basée sur la forte strati- 
fication verticale de densité que l'on rencontre couramment dans les lacs, ce qui facilite la tâche des modélisateurs, car cette structure particulière empêche les mouvements verticaux. En effet, la stratification thermique, même si elle est faible, représente une grande quantité d'énergie potentielle qui stabilise la masse d'eau. Les perturbations turbulentes sont réduites à des épisodes intermittents qui induisent des mouvements horizontaux (coutants de densité, ondes internes) dont le rôle est de redistribuer les instabilités locales tout en conservant la structure unidimensionnelle. II suffit alors de représenter l'effet de ces phénomènes sur la structure verticale: les variations sur la verticale étant prédominantes dans les équations de bilan de masse, moment et énergie (Patterson et al., 1984), les variations transversales ou longitudinales y jouent un second rôle permettant de les négliger, ou de représenter avec des modèles du premier ordre les processus bidimensionnels comme les courants induits par le vent (Spigel et Imberger, 1980) ou les entrées et sorties d'eau (Ryan et Harleman, 1971; Hebbert et al., 1979; Akiyama et Stefan, 1984; Hocking et al., 1988; Imberger et Patterson, 1989).

Ces modèles sont donc surtout adaptés à la représentation de lacs de petite ou moyenne taille pour lesquels l'influence de la rotation de la terre (Force de Coriolis), des grandes circulations induites par le vent ou des effets advectifs (entrées et sorties d'eau) sont faibles en comparaison de l'influence de la stratification.

Nous allons présenter ici le modèle EOLE d'EDF-DER. C'est un modèle thermique unidimensionnel vertical qui existe depuis de longues années à EDF-DER, Département Environnement (Klein et Bonnaud, 1977; Enderlé, 1980) où il a servi de modèle de base pour de nombreuses études (Gaillard, 1981; Garçon, 1981; Enderlé, 1982; Enderlé et Gras, 1983; Enderlé-Salençon, 1983; Agoumi et al., 1985; Destuynder, 1986; Salençon, 1986; Salençon et Destuynder-Bélus, 1987 ; Gilbert et Salençon, 1988; Destuynder-Bélus, 1989; Salençon et Simonot, 1989; Pourcher et Salençon, 1990; Salençon et al., 1990; Salençon et Gilbert, 1991 ; Salençon, 1992). L'objectif de ce modèle est d'être couplé à un modèle biologique, donc d'être suffisamment robuste et prédictif, tout en restant simple, pour permettre de simuler l'évolution de l'écosystème pendant plusieurs cycles annuels (jusqu'à une dizaine) avec une échelle de temps journalière pour les processus biologiques, ce qui a orienté certains choix de modélisation que nous discuterons.

La dernière version de ce modèle a été développée dans le cadre de l'étude détaillée du réseau trophique de la retenue de Pareloup. Appliqué ensuite à des retenues de caractéristiques très différentes de celles de $\mathrm{Pa}$ reloup, il a permis d'obtenir de bons résultats. 


\section{DESCRIPTION DE LA RETENUE DE PARELOUP}

La retenue de Pareloup est située dans l'Aveyron, près de Rodez, sur le plateau du Lévezou dont l'altitude varie de 700 à $900 \mathrm{~m}$. Ce plateau, situé sur les premiers reliefs à l'Est du Bas$\sin$ Aquitain, est soumis à un régime climatique globalement océanique, caractérisant surtout le printemps et l'automne, avec une influence continentale en hiver et méditerranéenne en été. Les hivers longs et rigoureux (100 à 120 jours de gel par an) se prolongent au début du printemps. Les étés sont courts et frais, avec une température d'air moyenne dépassant rarement $20^{\circ} \mathrm{C}$. Les précipitations sont assez régulièrement réparties dans l'année, avec des orages l'été et un enneigement important l'hiver en raison de l'altitude. Le plateau du Lévezou est venté une grande partie de l'année. On peut distinguer les vents violents froids et secs qui soufflent I'hiver du secteur Nord-Nord-Est, les vents humides océaniques d'OuestNord-Ouest dominants au printemps et à l'automne et les vents chauds et humides de Sud-Sud-Est, responsables des orages d'été. On peut dire que, dans son ensemble, le Lévezou a un climat rude.

L'aménagement du Pouget, qui utilise le dénivelé existant entre le plateau du Lévezou et le sillon creusé par le Tarn, $500 \mathrm{~m}$ plus bas, est organisé autour du lac de Pareloup, réservoir culminant qui occupe une dépression favorable sur le cours du Vioulou (fig. 1). La très grande capa- cité de cette retenue $\left(169 \mathrm{hm}^{3}\right)$, lui permet d'emmaganiser l'eau pendant les saisons de moindre besoin en électricité, pour l'utiliser pendant les saisons de forte demande (principalement l'hiver). La cote du plan d'eau reste haute et stable en été, pendant la période touristique.

Outre les apports naturels du Vioulou, de la Gourde et du Ceor (débit moyen annuel de $3,3 \mathrm{~m}^{3} \cdot \mathrm{s}^{-1}$ ), la retenue reçoit l'eau des bassins versants du Viaur et du Bage, par l'intermédiaire des retenues de Pont de Salars $\left(20 \mathrm{hm}^{3}\right)$ et de Bage $\left(4 \mathrm{hm}^{3}\right)$, qui fonctionnent en vases communicants. La station de pompage de Bage (débit maximum de $14 \mathrm{~m}^{3} \cdot \mathrm{s}^{-1}$ ), remonte ces apports sur les $98 \mathrm{~m}$ de dénivellation séparant Bage de Pareloup avec un débit annuel moyen de $4,2 \mathrm{~m}^{3} \cdot \mathrm{s}^{-1}$. La conduite de Bage, débouche dans le lac à la cote 768 NGF'.

Dans la partie aval de l'aménagement, l'eau stockée dans Pareloup est turbinée à l'usine d'Alrance (chute de $80 \mathrm{~m}$ ) puis, après avoir transité par le réservoir de Villefranche de Panat, elle est restituée au Tarn à l'usine du Pouget qui exploite une chute de $461 \mathrm{~m}$. Une station de pompage au Pouget (débit maximum de $6,6 \mathrm{~m}^{3} \cdot \mathrm{s}^{-1}$ ) permet de remonter les eaux du Tarn aux heures creuses pour les stocker dans le réservoir de Villefranche de Panat.

Le lac de Pareloup est composé d'un corps principal orienté Est-Ouest correspondant à la vallée du Vioulou sur lequel est construit le barrage et

(־) NGF : Niveau Général de la France. 


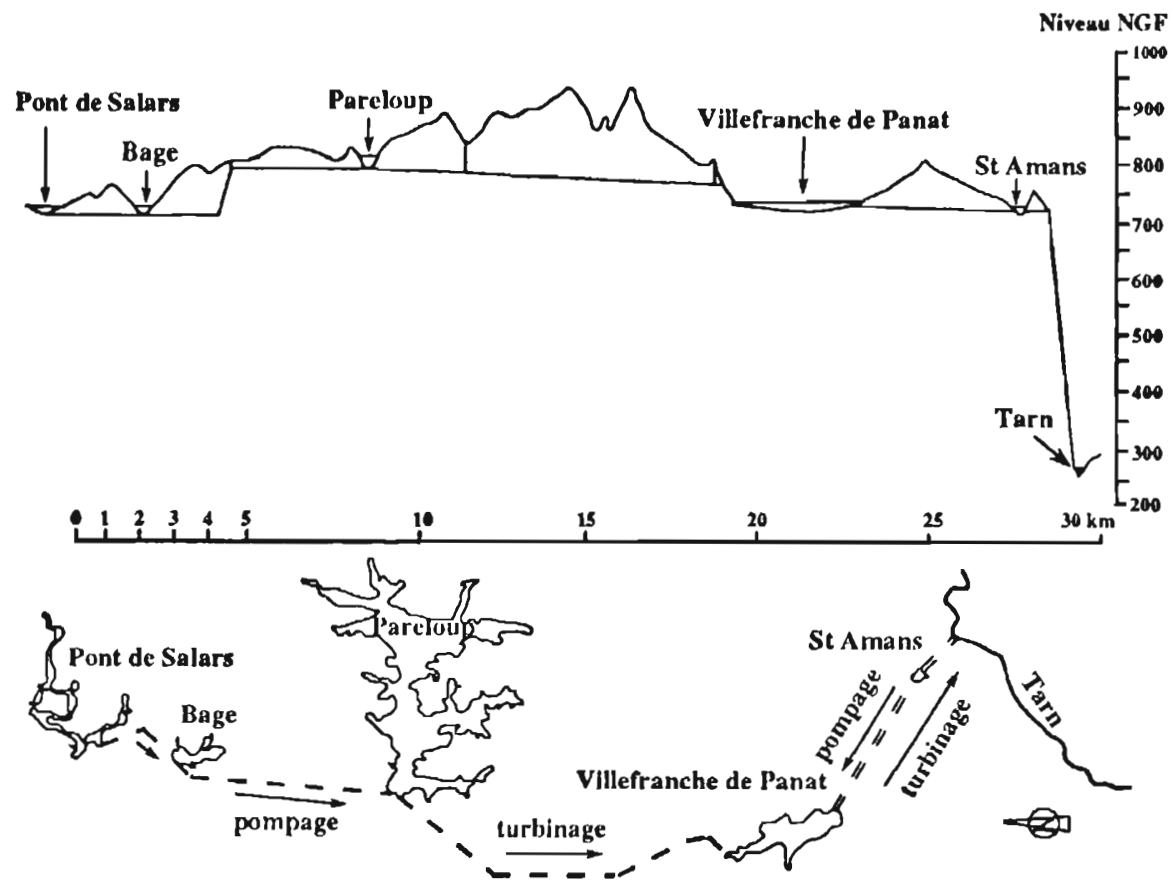

Fig. 1. - Profil en long général du complexe hydro-électrique de Pareloup (d'après EDF-GRPH Languedoc).

Fig. 1. - Schematic presentation of the Pareloup hydroelectric system. (from EDF-GRPH Languedoc).

de bras latéraux dont les 3 principaux sont situés au Sud et orientés NordSud (fig. 2).

A la cote maximale (805 NGF), sa capacité totale est de $169 \mathrm{hm}^{3}$ et la superficie de $12,6 \mathrm{~km}^{2}$. La profondeur maximale de la retenue à proximité du barrage est de 40 mètres. Elle diminue rapidement à 25 mètres au droit de l'ancien lit du Vioulou. L'essentiel du volume de la retenue est situé en surface, la capacité utile entre les cotes 775 et 805 NGF représentant $167,7 \mathrm{hm}^{3}$, la profondeur moyenne étant de $12,5 \mathrm{~m}$ et le centre de volume du lac étant situé à $9,75 \mathrm{~m}$ (fig. 6).
Les sorties d'eau de Pareloup sont de deux sortes: le débit réservé du Vioulou, restitué par une vanne en pied de barrage, et le débit turbiné. Le débit rẻservé, prélevé à la cote 764 NGF, est faible $\left(103 \mathrm{l} . \mathrm{s}^{-1}\right)$, alors que le débit turbiné prélevé dans le lac par une tour dont l'ouverture est comprise entre les cotes 775 NGF et 784 NGF, peut atteindre $24 \mathrm{~m}^{3} . \mathrm{s}^{-1}$.

Son temps de séjour est assez élevé (300 jours), ce qui entraîne une stratification thermique marquée de la colonne d'eau de mi-juin à début octobre ainsi qu'en hiver, avec formation, certaines années, d'une couver- 


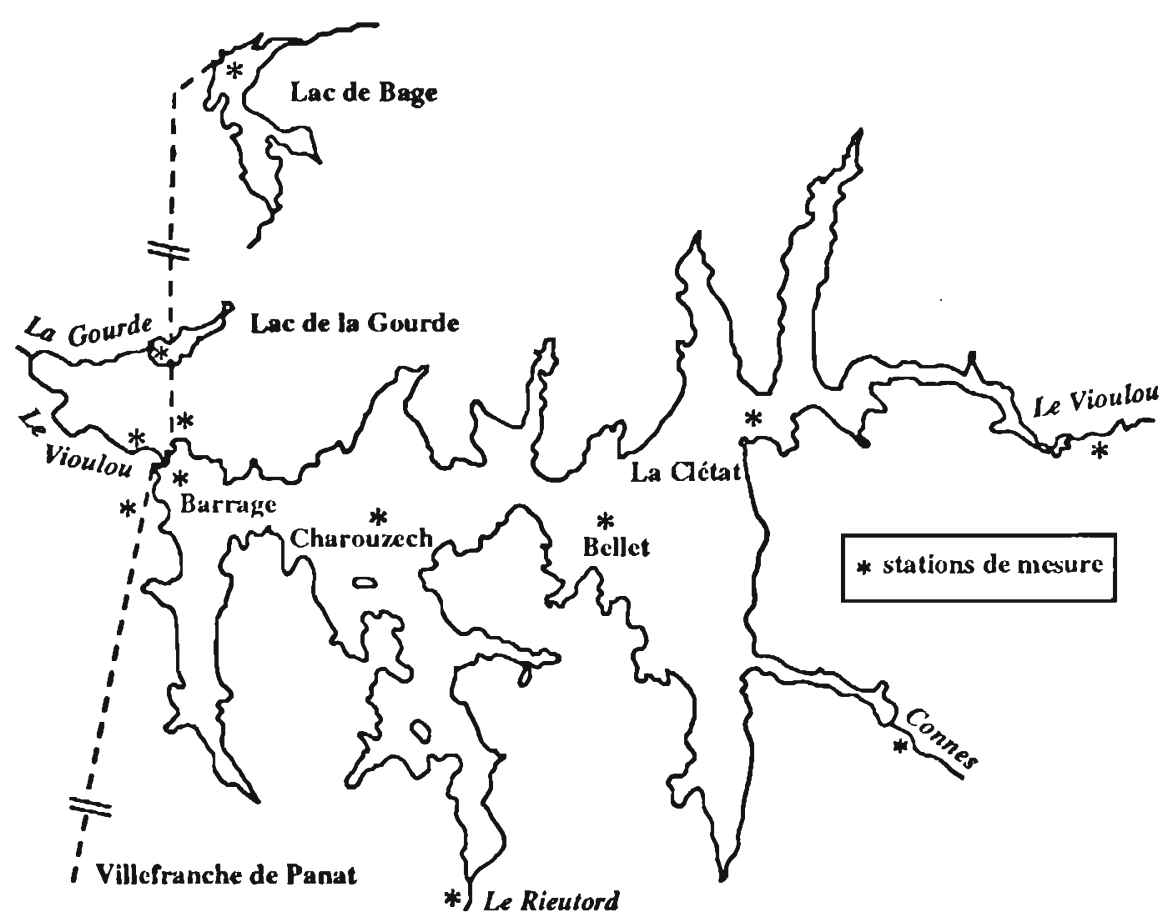

Fig. 2. - Plan des lacs de Pareloup, La Gourde et Bage.

Fig. 2. - Map of Pareloup, La Gourde and Bage lakes.

ture de glace: c'est un réservoir dimictique. Les mesures de concentration de chlorophylle a relevées depuis le début de l'étude classent cette retenue dans la catégorie des lacs mésotrophes.

On observe dès la fin du mois de février un développement de phytoplancton, essentiellement des diatomées dont le déclin, fin mai, coïncide avec l'apparition du gradient thermique. Cette phase d'eaux claires est associée à la chute des teneurs en $\mathrm{SiO}_{2}$ dans la zone euphotique, à la réduction de la turbulence qui favorise la sédimentation et au développement du zooplancton filtreur. Le peuplement d'été est moins dense mais plus diversifié. Lors de l'enfoncement de la thermocline en automne, on observe un nouveau développement de diatomées associées à quelques chlorophytes.

\section{STRUCTURE THERMIQUE D'UN RÉSERVOIR}

L'évolution de la structure thermique verticale d'un réservoir résulte des échanges atmosphériques et des apports et retraits d'eau au réservoir. 
Cette structure verticale est fondamentale pour l'écosystème, car elle est le traceur des mouvements internes des masses d'eau.

Dans nos régions tempérées, la plupart des réservoirs sont dimictiques, c'est-à-dire qu'ils sont complètement mélangés verticalement à l'automne et au printemps, et stratifiés en été et en hiver.

Au début de l'hiver, le lac est homogène : les eaux de surface se refroidissent en permanence au contact de l'atmosphère, deviennent plus lourdes et plongent, entraînant un mélange vertical par convection. Dès que la température hivernale de surface devient inférieure à $4^{\circ} \mathrm{C}$, température du maximum de densité de l'eau douce, une stratification thermique inverse s'installe : les eaux du fond restent à $4^{\circ} \mathrm{C}$, les eaux de surface se refroidissant progressivement jusqu'à la formation éventuelle d'une couche de glace en surface, si le refroidissement persiste. A la sortie de l'isothermie, au début du printemps, les couches de surface se réchauffent sous l'effet du rayonnement solaire, ce qui stabilise la masse d'eau en densité. Le vent fournit l'énergie nécessaire au mélange de cette eau chaude de surface jusqu'à une certaine profondeur. II y a alors création d'une légère stratification (thermocline transitoire), qui diminue considérablement les échanges verticaux. C'est de la dynamique printanière de cette stratification transitoire que dépend la structure thermique saisonnière. II est donc particulièrement important de bien la comprendre afin de pouvoir bien la simuler.

Dès que la retenue est stratifiée, les échanges thermiques intéressent uniquement la couche de surface et les eaux du fond de la retenue sont momentanément isolées. La couche de surface, du fait de son volume faible, s'échauffe rapidement jusqu'à atteindre un état d'équilibre avec les flux atmosphériques. A ce stade, l'accumulation de chaleur dans l'eau s'arrête. Elle ne peut reprendre qu'après un épisode de mélange qui détruit la thermocline transitoire, au cours d'épisodes de forts vents ou de refroidissement, et répartit cette chaleur dans tout le lac, ce qui permet d'abaisser la température de surface et entraîne un accroissement des flux à l'interface air-eau. L'alternance des phases de stratification et de mélange, qui a lieu habituellement au cours du cycle diurne, est un mécanisme particulièrement important car c'est lui qui permet, tout au long du printemps, le réchauffement progressif de la couche du fond au-dessus de sa température hivernale. Ainsi, lorsque les phases de stratification transitoire se prolongent plusieurs jours, l'accumulation globale de chaleur dans le lac est plus faible en raison de l'inertie moindre des masses d'eau de surface mises en jeu lors des échanges atmosphériques. Lorsque de telles situations se renouvellent souvent au cours du printemps, elles aboutissent à un réchauffement moindre de l'hypolimnion. 


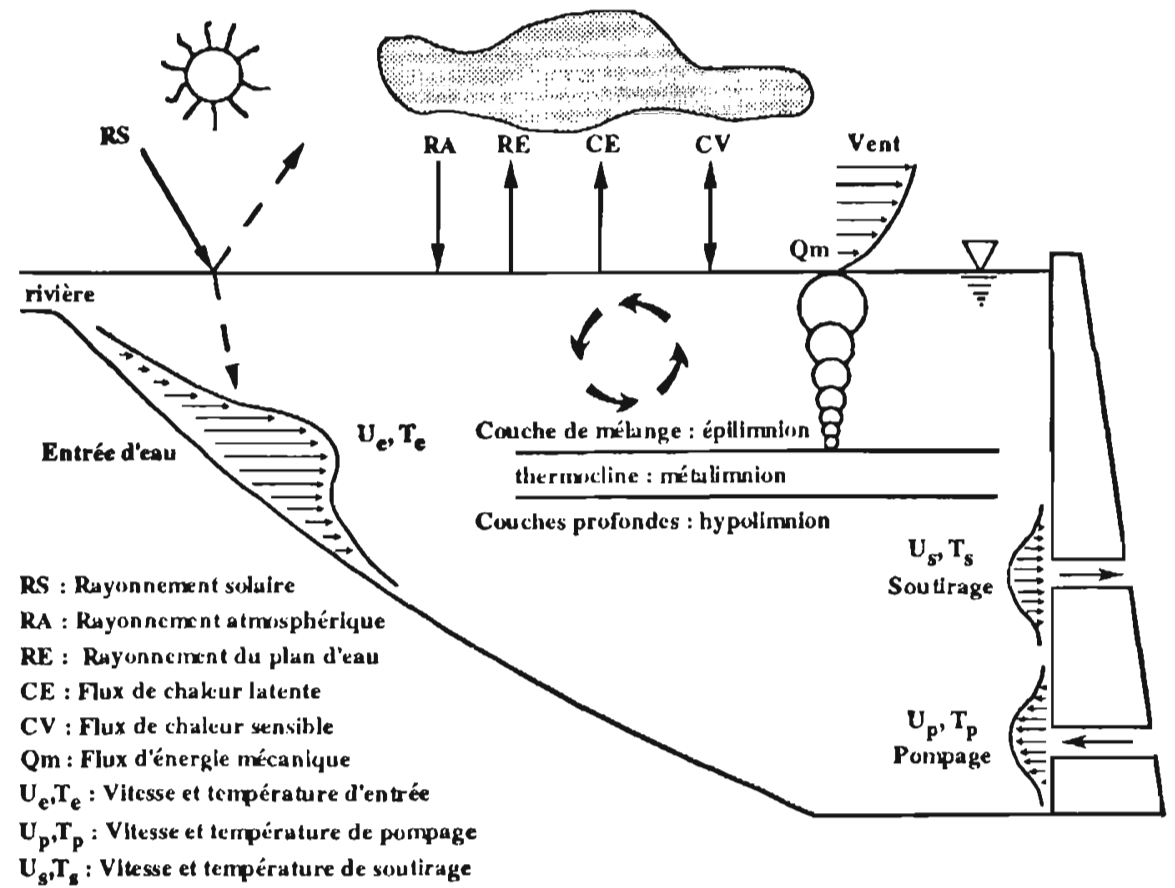

Fig. 3. - Bilan d'énergie d'une retenue et dynamique interne.

Fig. 3. - Energy budget and internal dynamics of a reservoir.

Vers la fin du printemps, l'énergie cinétique turbulente apportée en surface n'est plus suffisante pour aboutir à un mélange complet du lac. L'accumulation des thermoclines transitoires donne alors naissance à une zone de fort gradient thermique, la thermocline saisonnière, stable en profondeur. Le réservoir est schématiquement séparé en 3 zones (fig. 3 ) :

- la couche de surface turbulente et bien mélangée en contact permanent avec l'extérieur par l'interface air-eau, appelée épilimnion. Sa température est déterminée par les échanges atmosphériques.
- La zone de fort gradient thermique, la thermocline saisonnière ou métalimnion, qui se comporte comme une barrière de densité.

- Les couches profondes, appelées hypolimnion, qui sont séparées de l'épilimnion par la thermocline. Elles sont le siège de phénomènes diffusifs et d'épisodes ponctuels de mélange. La température des couches de fond est déterminée soit par la dernière phase du mélange hivernal soit par les entrées d'eau les plus froides. L'hypolimnion conservera cette température tout l'été, à l'abri de la thermocline saisonnière. 
A l'automne, le refroidissement des couches superficielles engendre des mouvements convectifs gravitionnels qui érodent la thermocline progressivement, jusqu'à obtenir le mélange hivernal. Les périodes de forts vents qui ont lieu à cette saison accélèrent ce processus de mélange.

Ces phénomènes très marqués dans un lac sont influencés par les phénomènes advectifs dans un réservoir. Les entrées d'eau se positionnent à une cote correspondant à leur couche de densité, entraînant avec elles des masses d'eau avoisinantes. Les soutirages mobilisent une couche horizontale du réservoir dont la hauteur dépend du débit et de la stratification thermique. Les mouvements horizontaux d'entrée et sortie d'eau ont pour effet de modifier la répartition verticale au sein de la masse d'eau.

\section{LE MODĖLE THERMIQUE UNIDIMENSIONNEL VERTICAL : EOLE}

\section{IV.1 Les équations du modèle thermique}

Une retenue d'eau peut être schématisée comme un fluide contenant un certain nombre de marqueurs (température, salinité, oxygène dissous, éléments nutritifs, phytoplancton, zooplancton etc.). "Etudier l'évolution d'un lac pendant un cycle annuel avec un modèle mathématique" se traduit, dans un modèle, par "Résoudre les équations non permanentes d'évolu- tion du fluide récepteur et des marqueurs, en se donnant des conditions initiales et des conditions aux limites".

Aux équations ponctuelles décrivant les mouvement du fluide (les équations dynamiques et l'équation de continuité), on rajoute, en général, l'équation de transfert thermique car la température détermine la densité de l'eau et la résolution des équations doit être couplée. II en serait de même pour tout traceur dont dépendrait la densité (salinité, matières en suspension etc.).

Selon les hypothèses classiques des modèles de turbulence (Niiler et Kraus, 1977), chaque variable $x$ est décomposée en une valeur moyenne $X$ et une fluctuation turbulente $X^{\prime}$, telles que $x=X+X^{\prime}$. L'opérateur de moyenne (noté ) est défini, pour l'échelle de temps $\tau$ par

$$
\bar{x}=X=\frac{1}{\tau} \int_{t-1 / 2}^{t+-/ 2} x d t
$$

et il apparaît donc que $\overline{X^{\prime}}=0$

Les équations locales instantanées sont alors moyennées sur la période de temps $\tau$, permettant d'obtenir un système d'équations de la turbulence établies localement en tout point $(x$, $y, z)$.

Selon le type de modèle retenu (modèle $0-D, 1-D, 2-D$ ) on procède ensuite à une opération de moyenne spatiale, selon $x$ et $y$ pour un modèle unidimensionnel, selon $y$ par un modèle bidimensionnel (Salençon et Simonot, 1989). Cette opération permet de tenir compte de la topographie. Parfois, lorsque la topographie varie 
Tableau 1. - Equations du modèle thermique unidimensionnel vertical intégrées en $\boldsymbol{x}$ et $y$.

Table I. - One-dimensional vertical thermal model equations integrated in the $x-y$ plane.

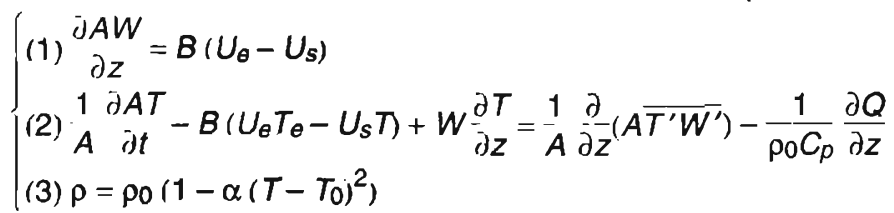
avec

$T(z) \quad$ la température de l'eau en ${ }^{\circ} \mathrm{C}$

$W(z) \quad$ la vitesse verticale en $\mathrm{m} \cdot \mathrm{s}^{-1}$

$A(z) \quad$ la surface horizontale du réservoir en $\mathrm{m}^{2}$

$B(z) \quad$ la largeur du réservoir en $m$

$U_{e}(z), U_{s}(z)$ les vitesses horizontales des flux entrants et sortants en $\mathrm{m} \cdot \mathrm{s}^{-1}$

$T_{\mathrm{e}} \quad$ la température de l'eau du flux entrant en ${ }^{\circ} \mathrm{C}$

$C_{p} \quad$ la capacité thermique massique à pression constante en $\mathrm{J} \cdot \mathrm{kg}^{-1} .{ }^{\circ} \mathrm{C}^{-1}$ $\left(C_{p}=4180\right)$

$Q(z) \quad$ le flux thermique dans la masse d'eau supposé uniquement vertical (pénétration du rayonnement solaire) en W. $\mathrm{m}^{-2}$.

L'équation d'état (3), proposée par Markofsky et Harleman (1971), permet de représenter le maximum de densité de l'eau à $4^{\circ} \mathrm{C}$, mais on suppose l'influence de la salinité et des matières en suspension négligeable.

$\rho_{0}=10^{3} \mathrm{~kg} \cdot \mathrm{m}^{-3}$

$T_{0}=4{ }^{\circ} \mathrm{C}$

$\alpha=6.6310^{-6}{ }^{\circ} \mathrm{C}^{-2}$

peu, il sera plus simple de ne pas l'inclure dans le modèle.

Dans le cas du modèle unidimensionnel vertical, il n'y a pas de modèle de circulation. L'intégration de ces équations sur les volumes finis des mailles du domaine de calcul, réalisée en utilisant la formule de Green, revient à faire le bilan des flux qui entrent et sortent par les parois de la maille (Chabard et al., 1989). Seules interviennent les conditions aux limites amont et aval de la composante horizontale, déterminées par le flux entrants $U_{\mathrm{e}}(z)$ et sortants $U_{\mathrm{s}}(z)$. Nous nous limiterons ici à présenter le système d'équations à résoudre (tableau I), le détail de ces opérations mathématiques étant décrit par Salençon (1994).

En appliquant l'approximation hydrostatique de Simons (1980) rappelée par Salençon et Thébault (1994b), nous pouvons utiliser la méthode des pas fractionnaires (Yanenko, 1968) pour résoudre le modèle global en un sous-modele vertical et un sous-modèle horizontal. 


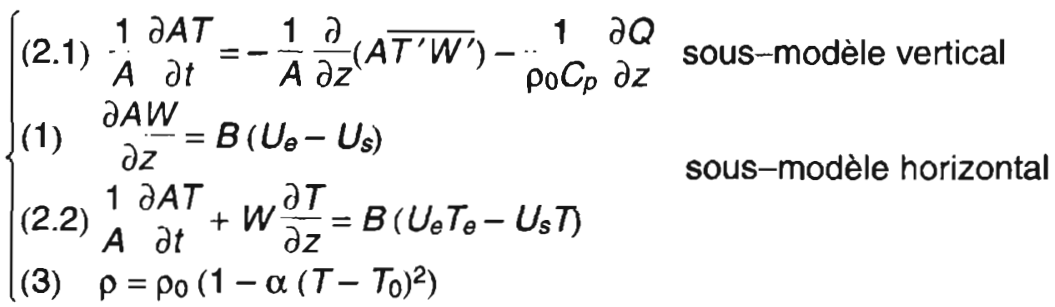

Nous insisterons particulièrement sur la description du sous-modèle vertical qui est fondamental dans la simulation de la stratification thermique d'un plan d'eau. Le sous-modèle horizontal est décrit en détail par Salençon (1994).

\section{IV.2 Le sous-modèle vertical}

Construire un modèle thermique unidimensionnel vertical consiste à résoudre l'équation de bilan thermique suivante :

$\frac{\partial T}{\partial t}=-\frac{1}{A} \frac{\partial}{\partial z}\left(\overline{A T^{\prime} W^{\prime}}\right)-{ }_{\rho_{0}} C_{p}^{-} \frac{\partial Q}{\partial z}$

variation transport source locale

temporelle $\begin{array}{ll}\text { vertical } & \text { de chaleur } \\ \text { (rayonnement solaire) }\end{array}$

où la variation temporelle de la température $T$, est le bilan entre un terme source et un terme de transport turbulent vertical.

Une des difficultés majeures dans l'étude hydrodynamique des lacs consiste à incorporer les phénomènes de transports turbulents, responsables de la stratification thermique. Faisant suite au bref historique de Harleman (1982), une revue très complète de l'évolution des modèles unidimensionnels de stratification thermique dans les lacs a été réalisée plus récemment par Imberger et Patterson (1989).
Nous rappellerons les deux types de modèles essentiellement utilisés pour résoudre les équations de transports turbulents: les modèles à coefficients de diffusion et les modèles à couche de mélange.

\section{IV.2.1 Les modèles à coefficients de diffusion et longueur de mé- lange}

Cette méthode, basée sur l'analogie avec les transports moléculaires, suppose que les flux turbulents peuvent s'exprimer par le gradient de la quantité transportée multíplié par un coefficient de diffusion turbulente.

$$
\frac{\partial}{\partial z}\left(\overline{T^{\prime} W^{\prime}}\right)=\frac{\partial}{\partial z}\left[\left(\alpha+K_{z}\right) \frac{\partial T}{\partial z}\right]
$$

$\alpha$ : coefficient de diffusion moléculaire $K_{z}$ : coefficient de diffusion turbulente

Cette méthode se heurte à la difficulté de définir les coefficients de diffusion turbulente qui sont des fonctions de l'espace et des conditions locales de stabilité. Cette modélisation a été historiquement développée en premier avec des coefficients constants déterminés par ajustement des calculs à des séries de profils thermiques mesurés (Ryan et Harleman, 1971). Des développements ultérieurs ont permis, à partir de considérations théoriques (modèles de turbulence d'ordre supérieur), d'in- 
corporer certains processus (stratification, effet du vent) avec une formulation de $K_{z}$ fonction du nombre de Richardson (Tassin, 1986). II reste cependant que, dans ce type de modélisation, la description globale du transport turbulent avec un seul coefficient de diffusion ne permet pas d'individualiser les processus générateurs de turbulence (vent, convection pénétrante, ondes internes, seiches, mélanges sur les bords...), d'identifier ceux qui sont dominants ni de représenter les interactions entre ces différents mécanismes (Harleman, 1982; Spigel et al., 1986). De plus, cette méthode est en défaut lorsqu'il n'y a plus de gradient thermique.

\section{IV.2.2 Les modèles à couche de mélange}

Ces modèles ont été développés initialement pour l'étude des couches superficielles océaniques (Niiler et Kraus, 1977). Ils sont basés sur l'observation que la couche superficielle est pratiquement bien mélangée. La répartition verticale de température, salinité et vitesse horizontale est pratiquement homogene et les variations y sont, de toute façon, négligeables par rapport aux variations aux limites ou à travers la thermocline. II est alors possible d'intégrer verticalement les équations turbulentes sur la couche de surface, supposée homogène. La hauteur de cette couche de mélange devient alors une variable du système. Cette méthode permet d'exprimer les flux de transports turbulents en termes de quantités moyennes et de conditions aux limites (apports extérieurs).

La préoccupation des modélisateurs est alors de quantifier comment l'énergie cinétique turbulente augmente l'énergie potentielle de la colonne d'eau en entraînant de l'eau plus dense et non turbulente dans la couche de mélange pendant des périodes de vent ou de refroidissement. Il est alors nécessaire de faire intervenir l'équation de conservation de l'énergie cinétique turbulente, obtenue en faisant le produit scalaire de l'équation de conservation du moment par le vecteur vitesse $\bar{U}$ (Niiler et Kraus, 1977).

Cette équation, également intégrée sur la couche de mélange, décrit comment l'énergie cinétique turbulente produite en surface par les processus de mélange (cisaillement dû au vent, vagues, refroidissement atmosphérique...) est transportée (par les fluctuations des vitesses turbulentes) vers le bas de la couche de mélange et partiellement utilisée pour homogénéiser le fluide. A l'interface, l'énergie cinétique turbulente disponible, augmentée de celle qui est produite localement par cisaillement du courant moyen de part et d'autre de l'interface et diminuée de celle qui est dissipée par viscosité à petite échelle ou rayonnée par les ondes internes est transformée en énergie potentielle en entraînant, dans la couche de mélange, l'eau des couches inférieures.

Ces modèles, comme le remarquent les précurseurs Niiler et Kraus (1977), grâce à l'approche intégrale, s'appuient sur de bonnes bases phy- 
siques et aboutissent à des outils relativement simples et fiables, ce qui justifie le choix qui en est fait actuellement par un certain nombre de modélisateurs (Stefan et Ford, 1975; Hurley Octavio et al., 1977; Enderlé, 1982; Harleman, 1982; Spigel et al., 1986; Imberger et Patterson, 1989; Salençon 1994).

Dans ces modèles, les mécanismes physiques qui déterminent la structure verticale sont représentés individuellement. Leur succès réside dans le fait que tous les processus dominants sont pris en compte. Ils permettent alors de bien comprendre la dynamique interne d'un lac et d'analyser l'effet de chaque mécanisme ou de l'interaction de plusieurs d'entre eux sur la structure verticale. Les valeurs des paramètres concernant les processus peuvent être estimées à partir d'expériences au laboratoire ou de mesures de terrain (Patterson et al., 1984).

En s'affranchissant du calage des coefficients liés à un lac particulier, ces modèles ont donc l'avantage de pouvoir être appliqués à différents lacs sans trop de variations des paramètres, les effets locaux étant pris en compte par la géométrie, les conditions aux limites et le forçage (météorologie, gestion hydraulique). Une mauvaise représentation du modèle (en comparaison avec les observations) doit être attribuée davantage à un mécanisme dominant n'ayant pas été pris en compte qu'à un problème de calage (Imberger et Patterson, 1989).
Ils sont donc adaptés pour être utilisés dans la compréhension de la réponse d'un lac, mais aussi comme outil prédictif, lorsque les conditions hydrométéorologiques varient et même dans le cas extrême de lacs en projet.

\section{IV.3 Le modèle hydrodynamique et thermique : EOLE}

Le modèle hydrodynamique et thermique EOLE décrit l'évolution de la stratification thermique saisonnière dans une retenue. Un grand soin doit être apporté à cette simulation, car la structure thermique verticale d'une masse d'eau est un traceur de ses mouvements internes, en relation directe avec les conditions hydrométéorologiques.

Le modèle utilisé ici est un modèle unidimensionnel vertical, basé sur l'hypothèse d'homogénéité horizontale. C'est un modèle intégral à bilan d'énergie, élaboré avec les équations classiques des modèles à couche de mélange (Niiler et Kraus, 1977; Harleman, 1982; Rayner, 1980; Spigel et al., 1986). Dans ce modèle, nous avons choisi de résoudre les équations intégrées en $x-y$, tenant compte explicitement de l'effet de la géométrie dans le système d'équations par le biais de la surface horizontale $A(z)$ du plan d'eau à la cote $z$, afin de tenir compte de l'effet de bord de la cuvette, particulièrement important dans le cas de masses d'eau fermées comme les lacs.

Nous avons choisi une discrétisation spatiale à volumes finis. Le lac 
est discrétisé en $n$ couches horizontales (numérotées de la surface vers le fond) d'épaisseur constante $\Delta z=$ $0,25 \mathrm{~cm}$. Seule la couche de surface peut avoir une épaisseur variable $\Delta z_{\mathrm{s}}$ $\leq \Delta z$ (fig. 4). Chaque couche $i$ est supposée homogène. La longueur du lac est supposée constante sur toute la profondeur. La largeur varie donc comme la surface. La surface de contact entre deux couches $i$ et $i+1$, notée $A(i+1)$, est calculée à partir des données bathymétriques (courbe surface - volume - profondeur). Les surfaces $A(i)$ sont interpolées linéairement en $z$ entre les valeurs de surface mesurées. Le volume de chaque couche $V(i)$ est calculé par intégration à partir des surfaces.

La résolution en temps est faite avec un pas de temps $\Delta t=3$ heures qui correspond au pas de temps de la saisie des données météorologiques.

Ce modèle étant présenté en détail par Salençon (1994), nous rappellerons simplement ici le principe du calcul.

A partir des échanges d'énergie à l'interface air-eau, un bilan énergétique est réalisé dans la colonne d'eau :

- bilan de l'énergie interne calculé à partir des flux de chaleur échangés à l'interface air-eau.

- bilan de l'énergie cinétique turbulente (ECT): l'ECT apportée en surface par le frottement du vent et la convection pénétrante crée une couche superficielle bien mélangée dont la hauteur est déterminée par le bilan entre cette énergie cinétique turbulente et la variation d'énergie potentielle de la couche de mélange.

Les mouvements des masses d'eau dus aux apports et soutirages d'eau dans la retenue sont représentés avec un modèle simple de transport, couplé au précédent. Les équations de continuité et de conservation de la chaleur sont intégrées sur un volume élémentaire et discrétisées dans l'espace et le temps (Enderlé, 1980).

Les entrées d'eau naturelles (rivières, apports du bassin versant...) dans le réservoir se positionnent à leur niveau de densité, entraînant avec elles des masses d'eau avoisinantes, alors que les entrées d'eau forcées (résurgences, injections d'eau à un niveau donné...) sont traitées en tenant compte du panache convectif qu'elles créent: une étude particulière, réalisée in situ, a permis de marquer les eaux pompées à Bage avec un colorant; ainsi individualisé le panache d'eau pompée a pu être suivi. Injectée dans le fond de la retenue, l'eau de Bage, après un mélange local, est restée dans l'hypolimnion en progressant horizontalement à son niveau de densité (Calmels et Salençon, 1991).

Dans le modèle EOLE, l'entrée du pompage a été représentée avec une distribution gaussienne du profil des vitesses horizontales, centrée sur le niveau où la conduite débouche dans le lac. La stabilisation verticale en densité des profils est ensuite assurée par un rattrapage convectif à partir du fond (Salençon, 1994). 
Débit d'eau entrant dans le réservoir

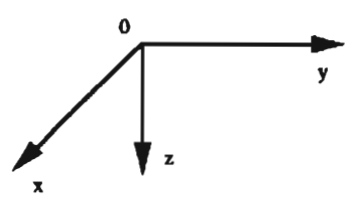

surface du reservoir

Qe

Débit d'eau sortant du réservoir au niveau de la prise d'cau clémentaire
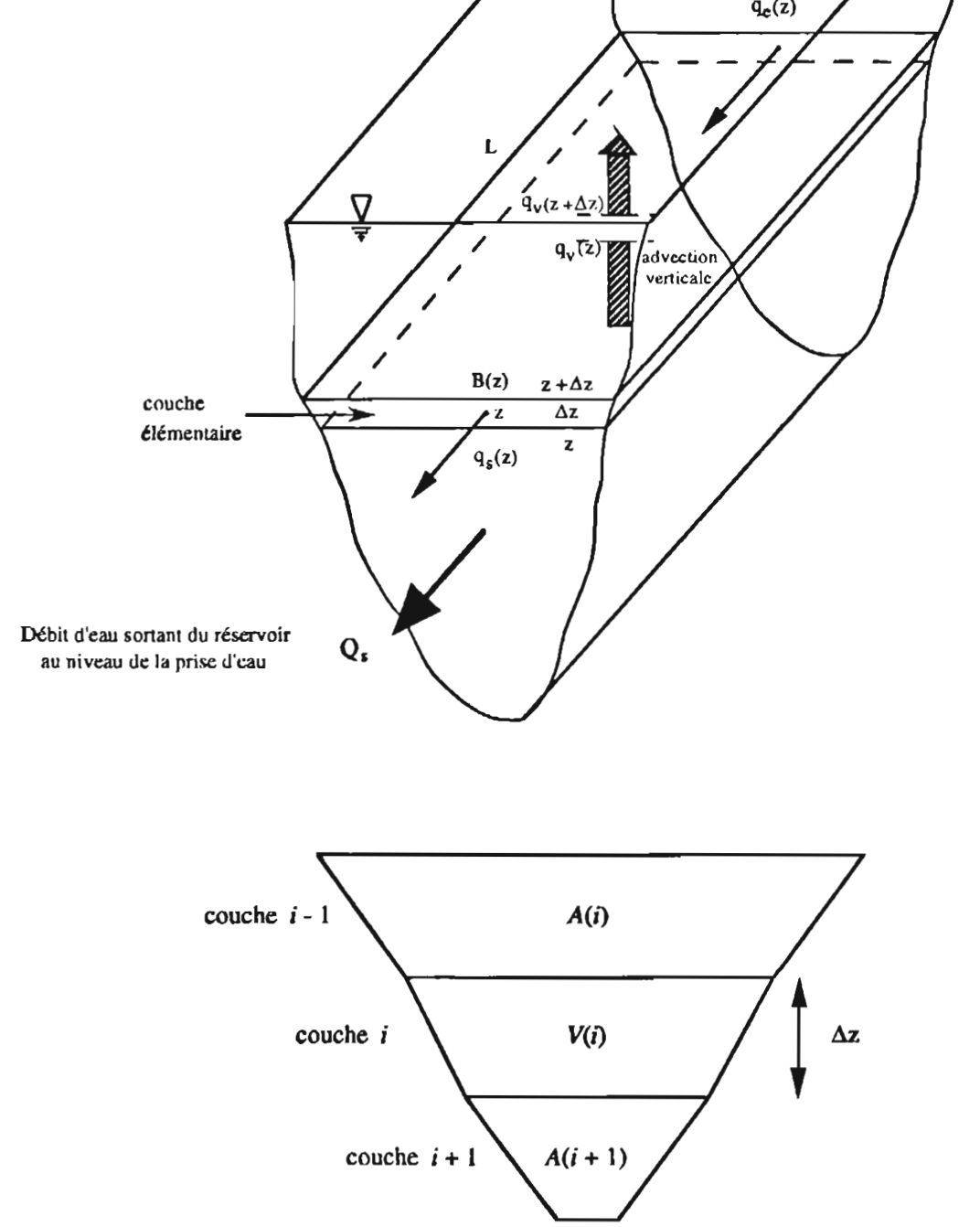

Fig. 4. - Discrétisation du réservoir en $n$ couches élémentaires d'épaisseur $\Delta z$.

Fig. 4. - Discretization of the reservoir into $n$ elementary layers with a thickness $\Delta z$ 
Les soutirages mobilisent une couche horizontale du réservoir dont la hauteur dépend du débit et de la stratification thermique. Les sorties d'eau sont centrées autour du niveau de soutirage.

Les vitesses horizontales d'entrée et de sortie d'eau sont distribués verticalement autour du niveau d'entrée ou de sortie selon un profil gaussien de vitesse qui représente l'entraînement des masses d'eau avoisinantes sous l'effet de jet (Markofsky et Harleman, 1971).

\section{APPLICATION DU MODĖLE EOLE À LA RETENUE DE PARELOUP}

Le modèle thermique EOLE a été appliqué à la retenue de Pareloup pendant une très longue période: 1976-1987.

\section{V.1 Les données d'entrée}

\section{V.1.1 Les données topographiques}

Dans le cas du modèle unidimensionnel, il suffit de connaître les courbes surface-profondeur et volume-profondeur pour dimensionner le maillage. Lorsque l'on étudie un retenue hydroélectrique, ces données font partie des archives de l'aménagement (fig. 5). Si l'on souhaite, comme à Pareloup, mettre à jour les données topographiques d'une retenue ancienne (en raison d'une sédimentation importante par exemple) ou si l'on étudie un lac naturel, il sera nécessaire de procéder à un levé bathymétrique, les surfaces étant calculées par planimétrage à partir des isobathes (Pujo, 1994).

\section{V.1.2 Les données météorologi- ques et hydrologiques}

Les données météorologiques trihoraires sont celles de la station du ré-

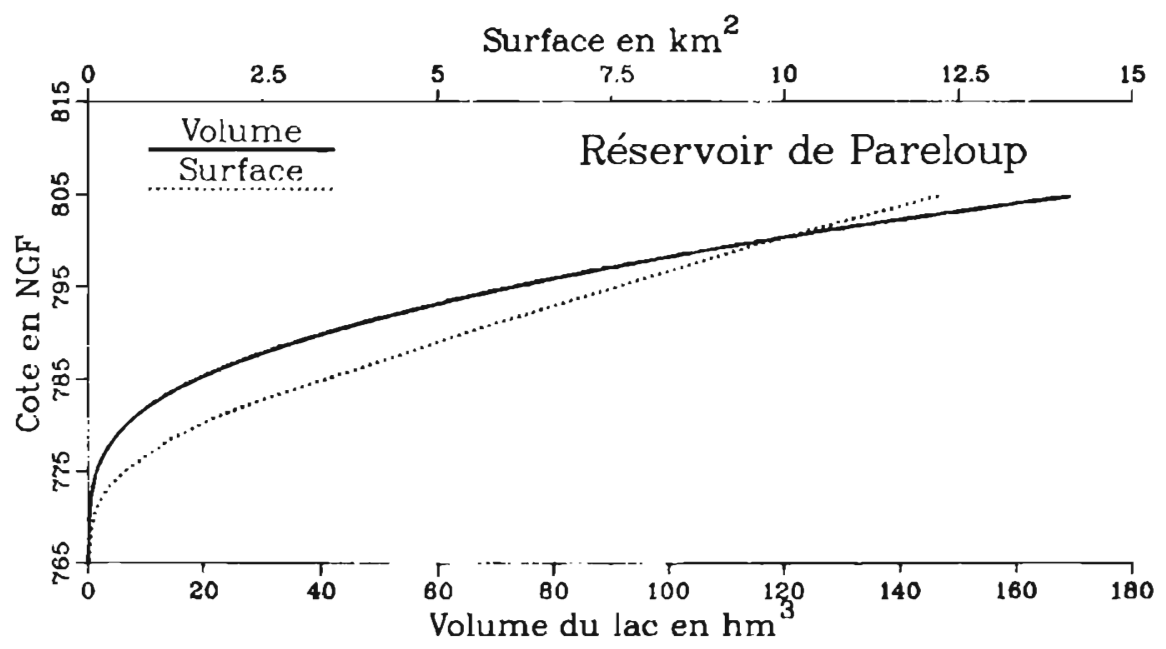

Fig. 5. - Courbes surface-profondeur et volume-profondeur du réservoir de Pareloup.

Fig. 5. - Surface-depth and volume-depth curves for Pareloup reservoir. 
seau national de Météo-France la plus proche, permettant de disposer de données de qualité pendant de très longues périodes. Lorsque cette station est éloignée du site, une station locale est implantée au bord du plan d'eau pendant la durée du suivi expérimental. Des corrélations faites entre les deux stations pendant les années de recouvrement permettent de déterminer, pour certains paramètres (vent, température d'air, pression atmosphérique, pluviométrie), une fonction de transfert qui sera ensuite appliquée aux données de la station du réseau si l'on souhaite disposer d'une chronologie plus longue. Pour la retenue de Pareloup, nous avons choisi la station de Millau, et une station météorologique locale a èté installée.

Les débits des rivières ainsi que les débits au travers des aménagements hydro-électriques sont fournis par les organismes nationaux en valeurs moyennes journalières.

\section{V.1.3 Le traitement des apports}

Dans un réservoir, la stratification thermique est influencée par les phénomènes advectifs. Les entrées d'eau s'insèrent à une cote correspondant à leur couche de densité, entraînant avec elles des masses d'eau avoisinantes. Ce mélange peut être important, en particulier lorsque le niveau d'insertion des entrées est imposé, comme dans le cas d'un pompage. Les mouvements horizontaux ont pour effet d'engendrer des mouvements verticaux qui ont tendance à lisser les profils verticaux.

Dans le cas d'un réservoir, il est donc particulièrement important de bien connaître tous les apports et leurs caractéristiques physico-chimi-

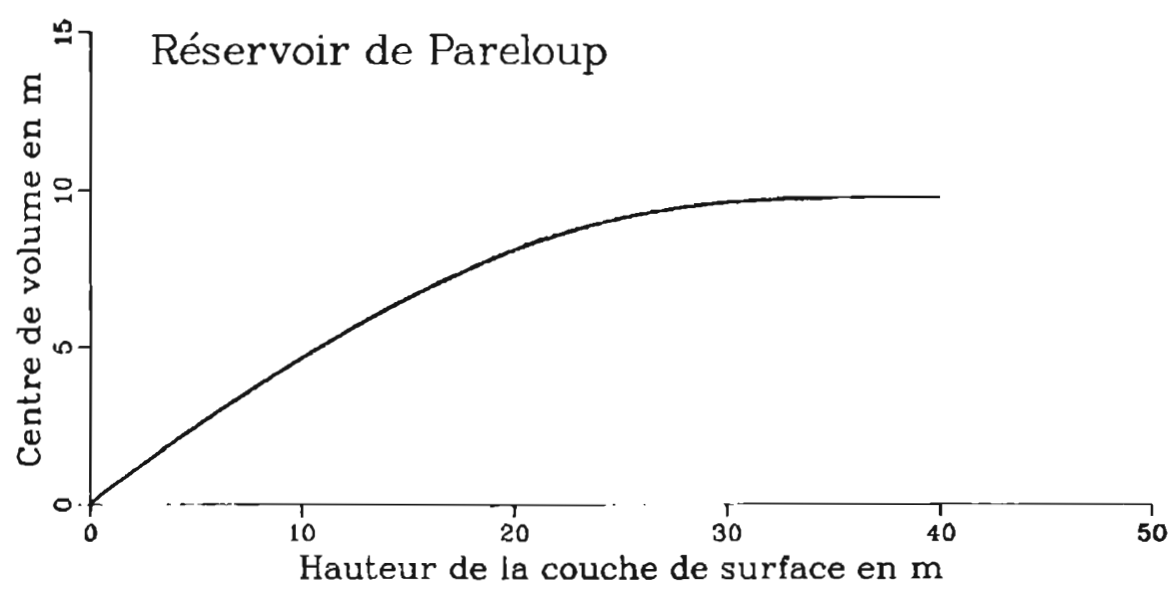

Fig. 6. - Retenue de Pareloup : centre de volume $L(z)$ d'une couche de surface de profondeur $z$. Fig. 6. - Pareloup reservoir: center of volume $L(z)$ of a surface layer at depth $z$. 
ques qui sont déterminantes dans le rôle qu'ils joueront pour l'écosystème.

Dans le modèle, les différentes entrées d'eau ont été regroupées en trois entrées de caractéristiques différentes:

- le pompage de Bage

- le ruisseau du Vioulou

- le ruisseau du Rieutord.

\section{V.1.3.1 Le bilan de l'eau}

De 1976 à 1987, un bilan d'eau a été calculé, chaque jour, à partir des mesures de débits entrants et sortants, et des relevés de cotes de la retenue. Ce bilan fait apparaître une sous-estimation des apports sur l'ensemble de la période étudiée, qui a été compensée par un débit correctif des apports (fig. 7).

Un tel écart peut avoir plusieurs causes:

- les différentes méthodes d'intégration de la courbe de capacité peuvent conduire à des estimations différentes du volume d'eau stocké. II ne faut pas négliger le fait que la courbe de capacité a été établie avant la construction du barrage en 1950, et que la bathymétrie a certainement évolué depuis.

- la précision des mesures de la cote du plan d'eau peut entraîner des erreurs considérables : en effet, pour une superficie de $12 \mathrm{~km}^{2}$, une erreur de $1 \mathrm{~cm}$ sur la cote correspond à un
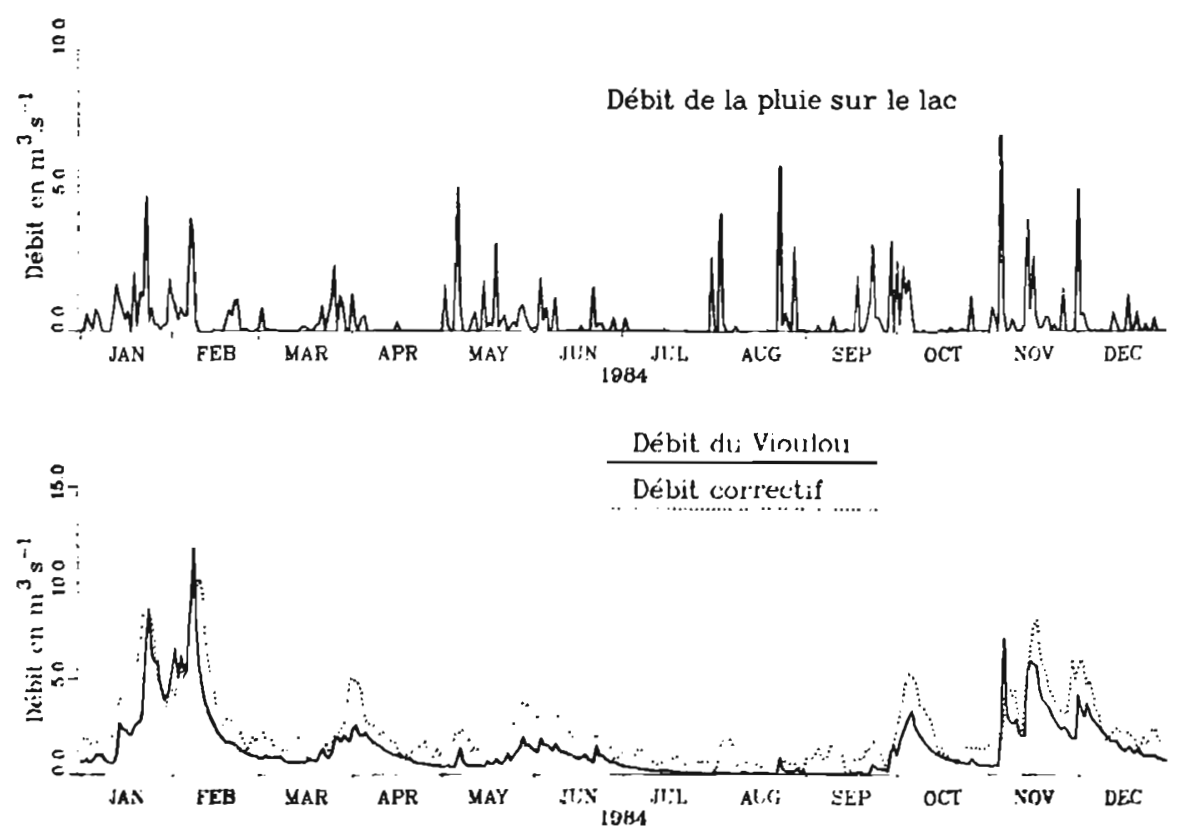

Fig. 7. - Bilan annuel de l'eau.

Fig. 7. - Annual water budget. 
débit moyen journalier de $1,5 \mathrm{~m}^{3} \cdot \mathrm{s}^{-1}$. Si les difficultés de lecture de l'échelle sont augmentées par les vagues, il ne faut pas non plus négliger les erreurs induites à la suite d'une inclinaison de la surface libre sous l'effet du vent (seiches-upwellings).

- les apports des ruisseaux qui n'ont pas été pris en compte, ainsi que les apports directs au lac (ruissellement sur les berges et précipitations à la surface du lac).

Comme le débit correctif est bien corrélé aux périodes de fortes précipitations ou de crues (sauf en été où le sol est $\mathrm{sec}$ ), cette dernière cause d'erreur paraît importante. Nous avons donc évalué le débit correctif comme la somme des apports des ruisseaux et des apports directs.

Le débit d'apport des ruisseaux non comptabilisés a été estimé à partir du débit mesuré du Vioulou et proportionnellement à la superficie de leurs bassins versants respectifs (fig. 8). Les apports directs ont été calculés avec les mesures pluviométriques journalières et les superficies des zones concernées.

Finalement, ces apports complémentaires ont été rajoutés au débit du Rieutord, dont les caractéristiques physico-chimiques paraissaient les plus proches de celles des petits ruisseaux.

\section{LAC DE PARELOUP}

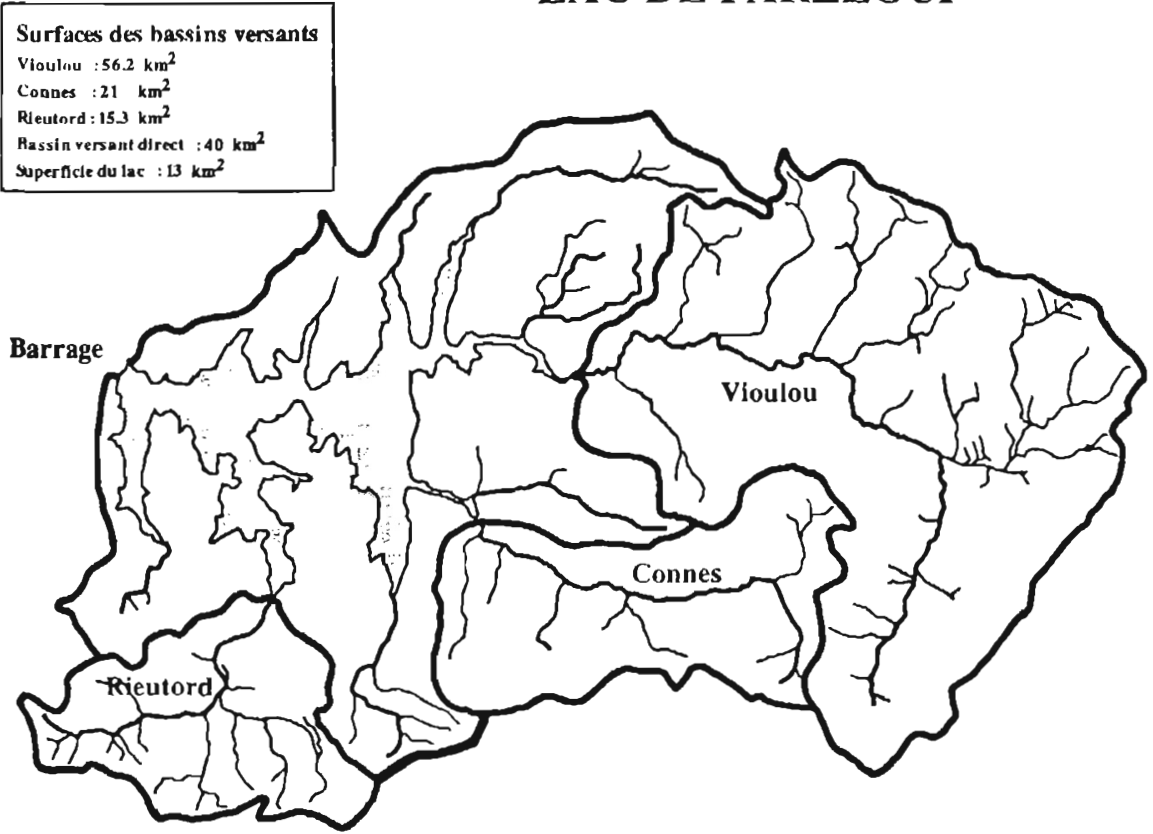

Fig. 8. - Le bassin versant de Pareloup (d'après Bosc, 1985).

Fig. 8. - The Pareloup watershed (after Bosc, 1985). 


\section{V.1.3.2 La température des apports}

II est particulièrement important de bien reproduire la température des apports car c'est elle qui détermine, par effet de densité, la profondeur à laquelle chaque entrée d'eau s'insère dans la retenue. Cette température n'étant pas toujours mesurée avec un pas journalier, les chronologies sont reconstituées par un calcul thermique calé sur les mesures disponibles.

Pour les affluents, les températures sont calculées avec un modèle thermique de rivière. Cette méthode a été mise au point lors de précédentes études sur de petits cours d'eau (Gilbert et al., 1986). Elle permet, de fa- çon simple, d'estimer les niveaux de température intermédiaires entre la température de la source, calculée par une température de nappe et la température à l'équilibre calculée avec le bilan de chaleur à l'interface air-eau. Elle consiste à appliquer un modèle de propagation d'une masse d'eau le long d'un tronçon de rivière à partir d'une température de nappe. Il est possible de tenir compte de la couverture végétale des rivières en réduisant le rayonnement solaire pendant la période de végétation. Ces calculs sont calés sur les mesures disponibles. Les résultats obtenus (fig. 9) montrent une bonne concordance entre calculs et mesures ce qui
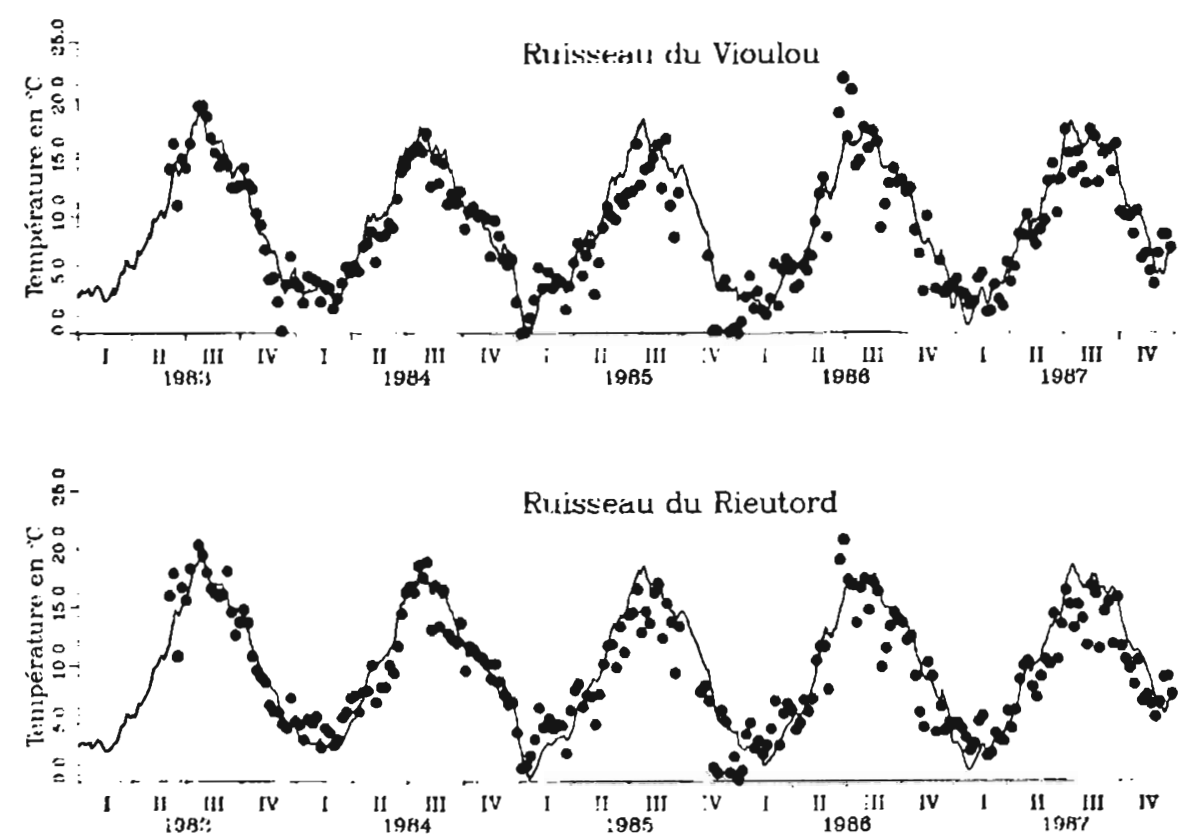

Fig. 9. - Reconstitution des chronologies de température des affluents. Chaque point correspond aux mesures: le trait continu correspond aux résultats de la simulation.

Fig. 9. - Reconstitution of affluent temperature chronologies. Each point corresponds to measurements; the solid line corresponds to simulation results. 

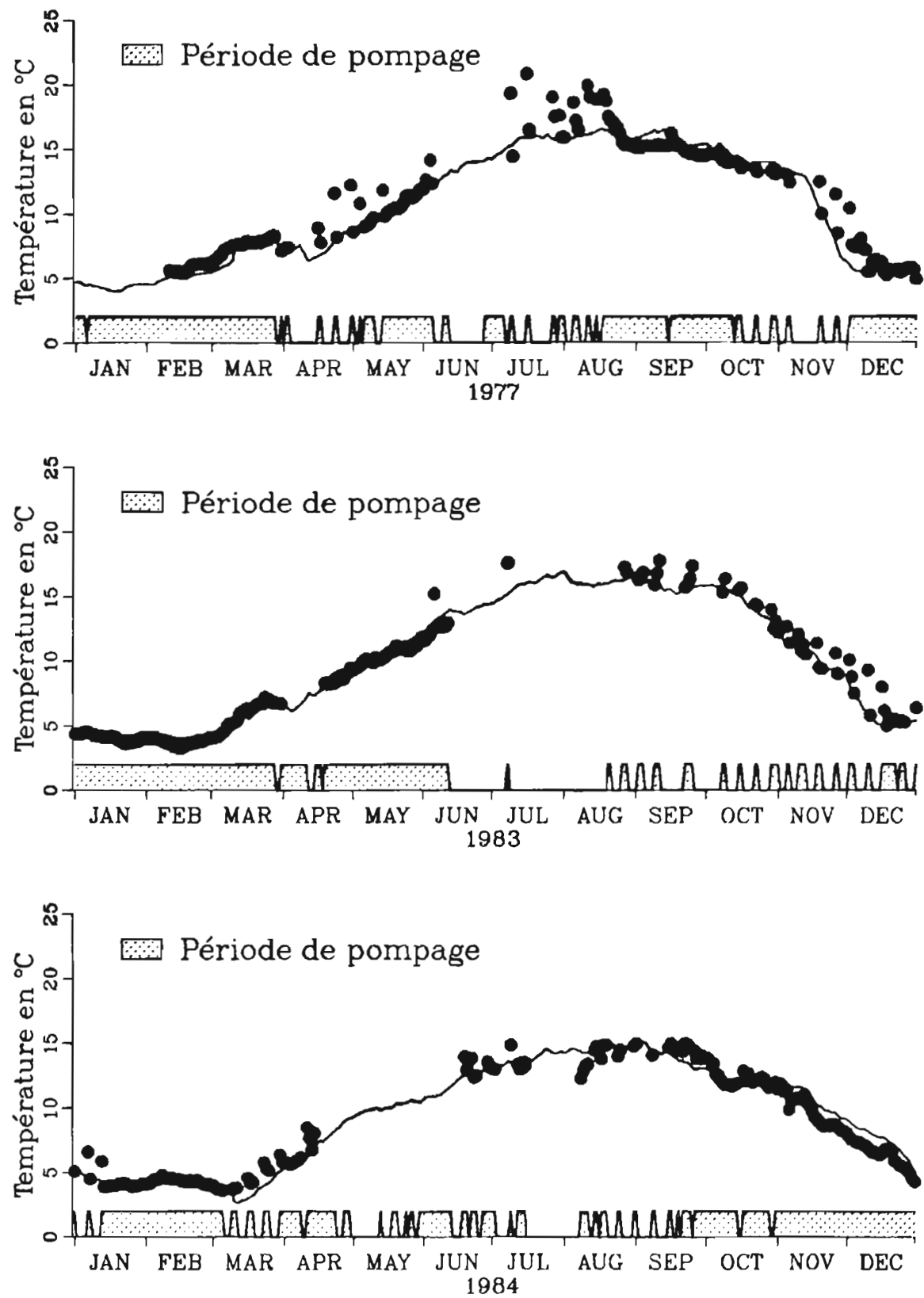

Fig. 10. - Calcul de la température de l'eau pompée dans la retenue de Bage. Chaque point correspond aux mesures; le trait continu correspond aux résultats de la simulation.

Fig. 10. - Calculation of the temperature of water pumped from Bage reservoir. Each point corresponds to measurements; the solid line corresponds to simulation results. 
permet, le modèle ainsi calé, de reconstituer les chronologies des températures pour toute la période d'étude.

La température de l'eau pompée dans la retenue de Bage a été calculée à partir d'une modélisation thermique de cette retenue. Les valeurs calculées journalièrement sont en bon accord avec les valeurs mesurées dans la galerie d'amenée lorsque le pompage est permanent (fig. 10). Lorsqu'il est intermittent, les températures mesurées sont plus chaudes car influencées par les apports gravitaires du petit lac de la Gourde qui, même s'ils sont faibles, ne sont plus dilués par le pompage.

\section{V.1.4 Le coefficient d'extinction}

Il est souhaitable de disposer de mesures du coefficient d'extinction avec un pas hebdomadaire pendant toute la durée d'un cycle annuel (fig. 11). Si ces mesures ne sont pas disponibles, une valeur moyenne annuelle peut être utilisée, mais il convient de procéder à une étude de sensibilité (Salençon et Gilbert, 1991).

\section{V.2 Les données de calage}

Pour le calage du modèle thermique, les profils thermiques ont été mesurés en plusieurs points du lac, toutes les trois heures, à l'aide de thermographes enregistreurs (11 capteurs thermiques répartis sur une verticale). Ces mesures ont été complétées par des profils hebdomadaires manuels au droit du barrage. Le suivi thermique de la retenue de Pareloup a dé- marré en 1976, mais, seule la période 1983-1987 ne comporte pas d'interruptions.

\section{V.3 Résultats du modèle thermique}

Le modèle thermique EOLE a été appliqué à la retenue de Pareloup pendant une très longue période: 1976-1987, pendant laquelle nous disposons de mesures. Nous allons analyser brièvement chaque année, en nous appuyant sur l'étude climatologique et hydrologique de la période 1976-1987.

\section{V.3.1 Etude climatologique et hydrologique de la période 1976- 1987}

A partir des données météorologiques de la station de Millau, disponibles de 1965 à 1987, des diagrammes ombrothermiques de Bagnouls et Gaussen (1953) ont été tracés. Ces diagrammes permettent de mettre en évidence les périodes de sécheresse, lorsque les courbes de température et de pluviométrie se recoupent. Le profil moyen de la période 1965-1987 (fig. 12) montre que les courbes ne se recoupent jamais.

Sur la même période, les débits transitant au travers de l'aménagement de Pareloup ont été analysés: débits d'entrée des ruisseaux, débits du pompage de Bage, débit du turbinage d'Alrance.

L'ensemble de ces résultats est résumé dans le tableau II.

Nous allons présenter maintenant une analyse exhaustive de chaque 
Retenue de Pareloup
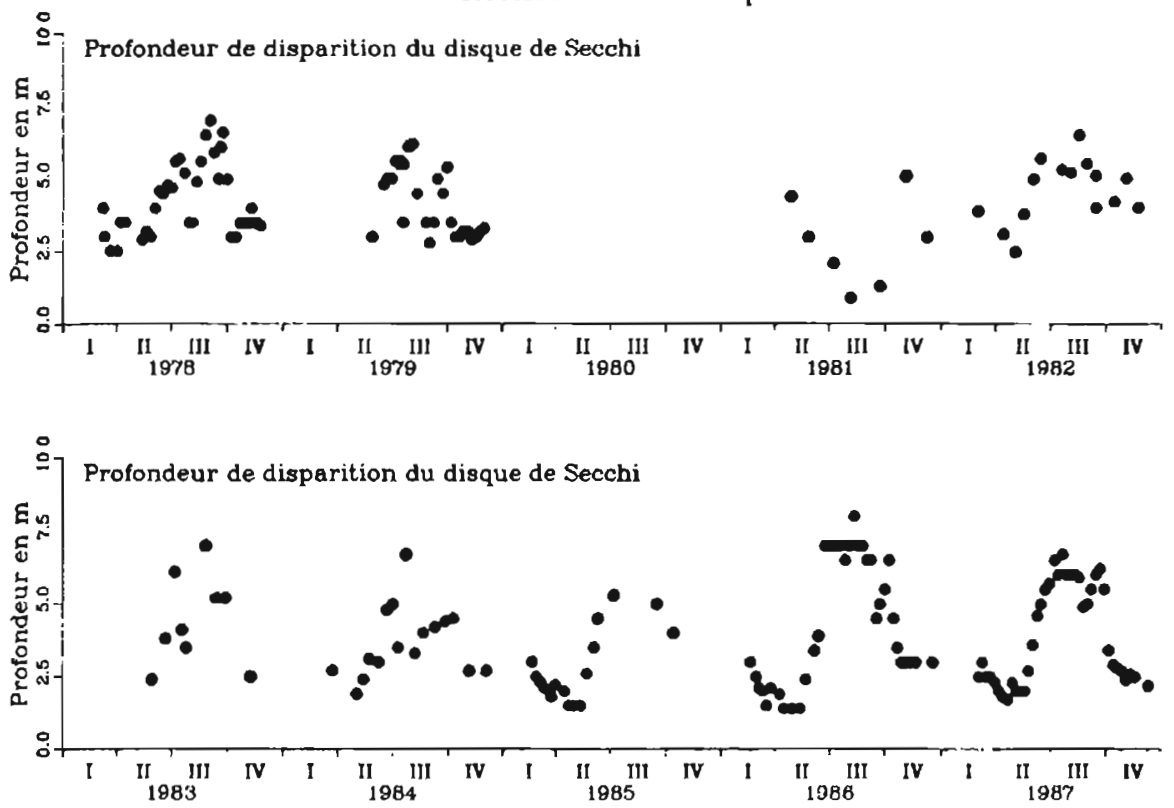

Fig. 11. - Profondeur de disparition du disque de Secchi mesurée dans la retenue de Pareloup de 1978 à 1987.

Fig. 11. - Measured depth at which the Secchi disk disappears in Pareloup reservoir, 1978 to 1987.

\section{Station météorologique de Millau Diagramme ombrothermique Période $1965-1987$}

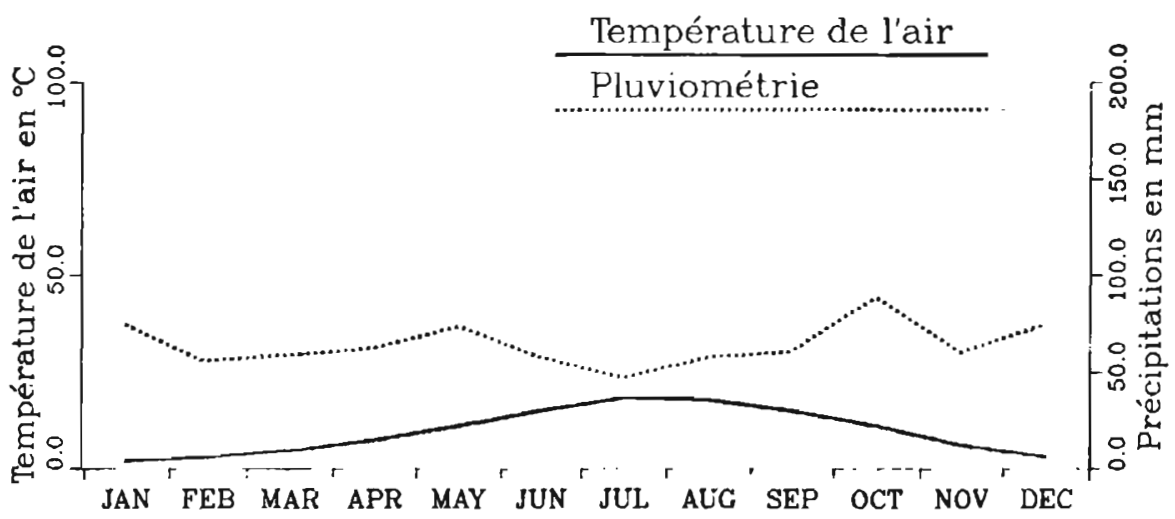

Fig. 12. - Diagramme ombrothermique à Millau 1965-1987.

Fig. 12. - Gaussen ombrothermic diagram for Millau, 1965-1987. 
Tableau II. - Etude climatologique et hydrologique de la période 1976-1987.

Table II. - Climatological and hydrological study of the period 1976-1987.

avril froid et humide juin chaud el très sec automne très pluvieux et frais mai sec

janvier à avril sec et doux mai à auût frais et pluvieux seplembre sec année fraiche

printemps pluvieux et froid juillct à novembre très sec

avril froid juin et juillet chaud et sec anût troid septembre sec octobre très pluvieux

janvier très froid

fevrier chaud printemps-élé froid pluviosité très imégulière

hiver froid

printemps (mars-avril) chaud mai pluvieux et froid juillet froid et humide auút sec
Tableau
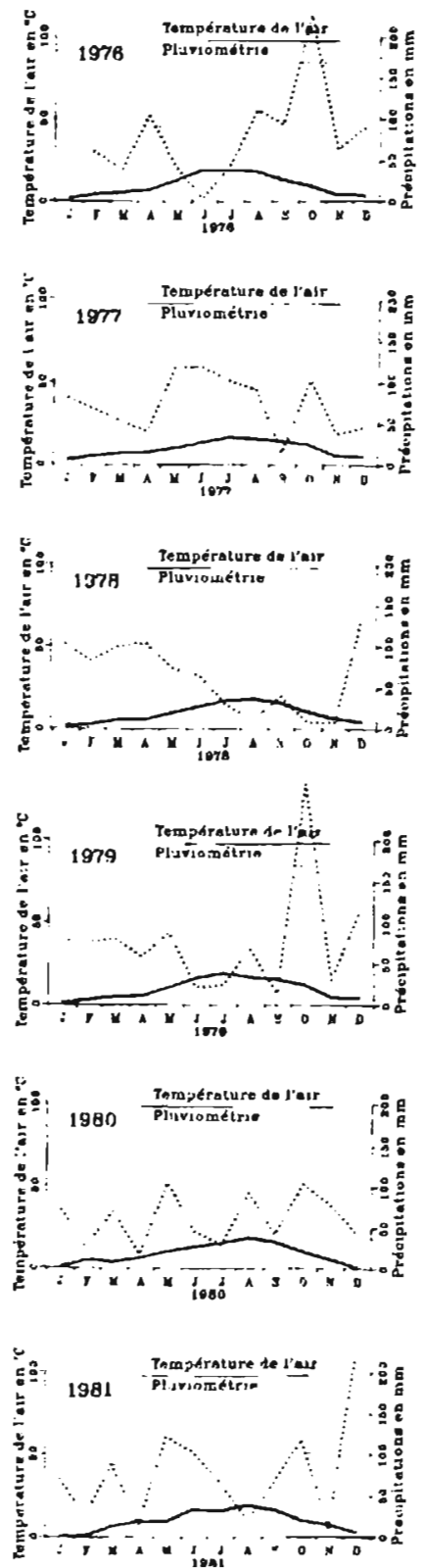

gestion très irrégulière

pompage quelques jours en juin-juillet de mi-aoút à mi-septembre pompage arrété de mi-septembre à mi-uctobre

turbine arrêtée de fin août à mi-novembre

plus de turbinage de mai à août plus de pompage de juin à août léger pompage cn avril-mai reprise de la gestion en octobre

gestion régulière toute l'année pas d'artêt l'étć (pluvieux)

gestion aclive jusqu’à fin mai arrêt en juillet el auût gestion faible en juin et automne-hiver

arrêt de la gestion de mars à mi-avril gestion faible mai-juin arrêt de la gestion de juillet à fin septembre

gestion continuc jusqu'à mi-juin arrêt du turbinage de mi-juin à début novembre

quelques jours de pompage fin juillet et en aoút 
Tableau II. - Suite

Table II. - Continued

hiver et printemps (janvier à mai) sec

juin-juillet chaud et humide automne-hiver pluvieux

févier très froid été et automne froid juillet sec et chaud
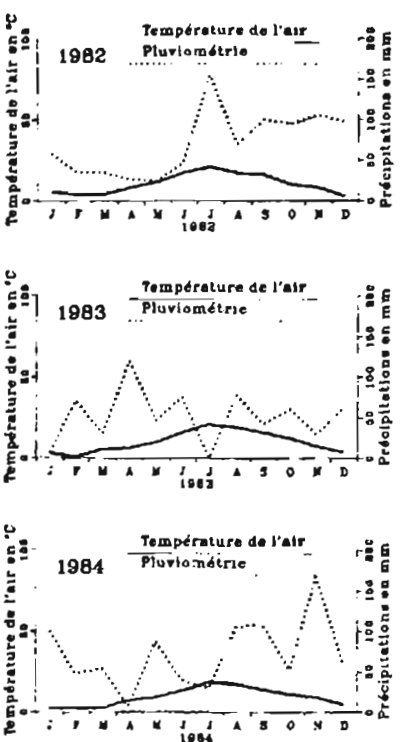

février à juillet froid sauf avri chaud et scc pluviosité irrégulière novembre chaud et humide juin-juillet sec août-septembre humide

janvier très froid printemps frais avec une pluviosité moyenne et régulière

juillet à octobre très sec et chaud

févricr et avril très froids printemps humide été (mai à auút) trés sec juillet à décembre chaud

janvier très froid printemps légèrement frais et sec jusquien juin avril chaud août et septembre secs et très chauds

octobre à décembre humide
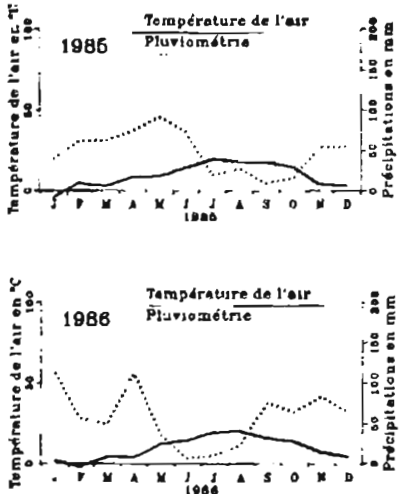

pompage presque continu jusqu'à fin mai de juin à fin août, pompage irrégulier. turbinage ancéte en septembre et octobre, pompage arrêté

gestion active en janvier et février gestion irrégulièrc en mars et août-sept. gestion arrêtée de mi-avril à mi-mai

et en juillet

reprise de la gestion en octobre

$$
\text { en septembre et } \propto \text { turbinage continu }
$$

pompage continu jusqu'à début mai pompage irtégulier en mai artêt du pompage pour travaux de juin à aoũt reprise du pompage irrégulière en septembre arrêt du pompage en octobre

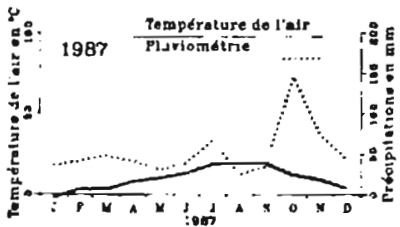

pompage continu jusqu'à fin avril arrêt complet de l'usinc de mai à aout 
année de la période 1976-1987. Une analyse plus synthétique de ces résultats est présentée au paragraphe V.3.14. Le lecteur non spécialiste peut se reporter directement au paragraphe V.3.14 sans dommage pour la compréhension générale.

\section{V.3.2 Année 1976}

Le début de l'année, de janvier à mars, a été sec, mais le mois d'avril a été froid et très humide. La gestion est très réduite à cette période et la retenue bien mélangée jusqu'à fin avril. En mai, la sécheresse et la grande chaleur débutent et se poursuivent jusqu'en juillet, pendant que la gestion est complètement à l'arrêt. La stratification démarre début mai, et reste installée jusqu'à fin septembre (fig. 14). L'arrêt complet de l'usine favorise une forte stratification, proche de celle d'un lac naturel: thermocline bien marquée, épilimnion chaud $\left(24^{\circ} \mathrm{C}\right)$ et hypolimnion froid $\left(8\right.$ à $\left.9^{\circ} \mathrm{C}\right)$. L'automne est très pluvieux et frais, ce qui permet une reprise de la gestion en octobre, qui accompagne un mélange automnal rapide. Cette année-là, la mise en place de la stratification est particulièrement bien reproduite par le modèle, confirmant que les mécanismes de mélange reliés aux échanges de surface sont bien pris en compte dans le modèle.

\section{V.3.3 Année 1977}

L'année 1977 est une année fraîche avec un été pluvieux pendant lequel l'usine a fonctionné régulièrement (fig. 13). La stratification s'établit tard, début juillet, lorsque la gestion hydraulique diminue. Aussi l'hypolimnion est chaud $\left(12^{\circ} \mathrm{C}\right)$, le gradient thermique de la thermocline faible et l'épilimnion relativement peu profond et
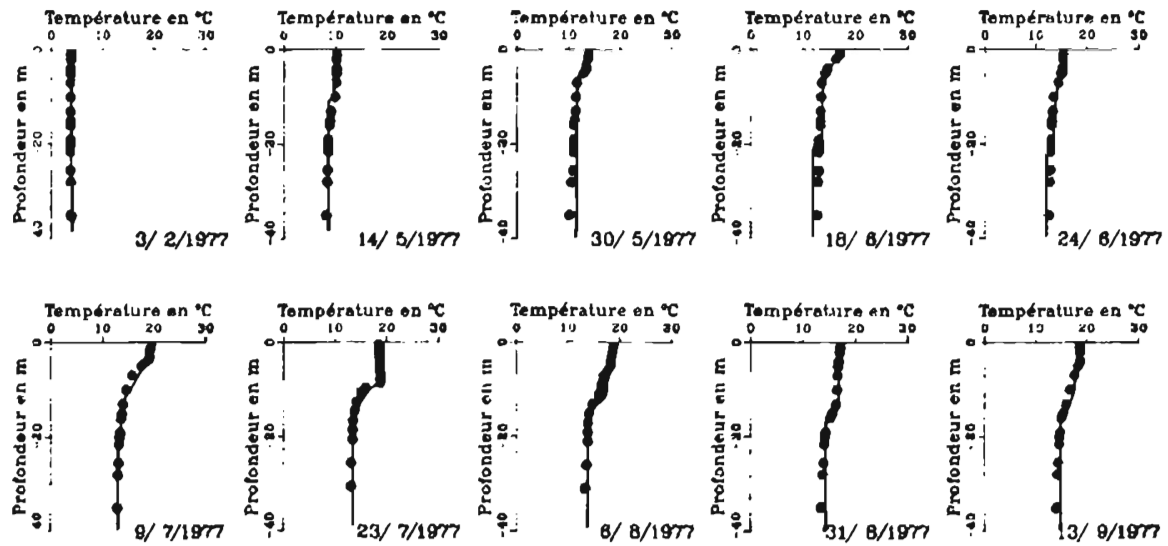

Fig. 13. - Retenue de Pareloup: comparaison des profils thermiques calculés (trait continu) et mesurés (•) en 1977. Le réservoir est tres peu stratifié, le printemps froid et pluvieux el l'été froid. Fig. 13. - Pareloup reservoir: comparison of calculated (solid line) and measured (•) thermal profiles in 1977. The reservoir is very little stratified, the spring cold and rainy, and the summer cold. 

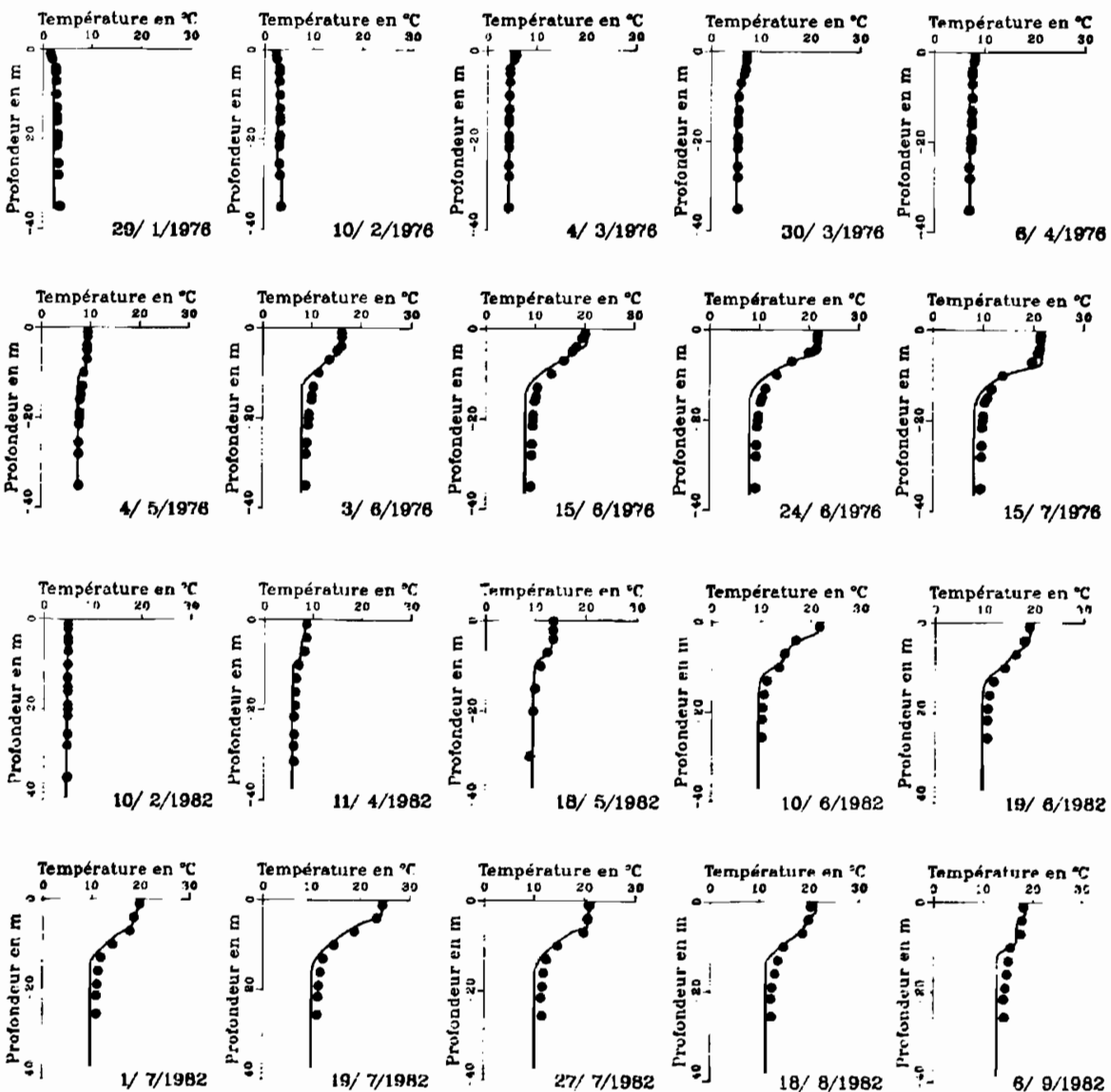

Fig. 14. - Retenue de Pareloup : comparaison des profils thermiques calculés (trait continu) et mesurés (•) en 1976 et 1982 . Le réservoir est très stratifié et l'été chaud.

Fig. 14. - Pareloup reservoir: comparison of calculated (solid line) and measured ( $\bullet$ ) thermal profiles in 1976 and 1982. The reservoir is highly stratified, and the summer hot.

frais (inférieur à $20^{\circ} \mathrm{C}$ en surface). Cette stratification peu marquée est très rapidement détruite dès fin septembre.

\section{V.3.4 Année 1978}

L'année 1978 a connu un printemps pluvieux et froid et une forte sécheresse de juillet à novembre (fig. 16). La gestion hydraulique, très active jusqu'à fin mai, s'est ralentie dès début juin jusqu'à la fin de l'année, avec un arrêt complet en juillet et août. La stratification apparaît fin mai. Dès que cesse le pompage en juillet s'établit un épilimnion chaud $\left(22^{\circ} \mathrm{C}\right)$ très près de la surface $(4-5 \mathrm{~m})$. Le gradient thermique est étendu sur une large zone (10 $\mathrm{m}$ environ) et la déstratification intervient rapidement fin septembre. 


\section{V.3.5 Année 1979}

En 1979, la gestion hydraulique a été très irrégulière et assez faible, avec un arrêt complet en mars et avril, ainsi que de juillet à septembre (fig. 18). La stratification est très marquée dès le mois de juin qui a été chaud. En juillet, l'épilimnion est profond (10-12 m) et, de ce fait, beaucoup plus froid qu'en $1978\left(19^{\circ} \mathrm{C}\right)$. II se réchauffe fin juillet $\left(23^{\circ} \mathrm{C}\right)$ sur toute sa profondeur, le mois de juillet ayant été chaud. Dès le mois d'août, qui est froid, débute la déstratification, avec le cisaillement du vent associé au refroidissement nocturne.

\section{V.3.6 Année 1980}

En 1980, le printemps présente des périodes chaudes et non ventées re- lativement longues, de l'ordre d'une semaine, favorisant l'installation d'une thermocline transitoire qui empêche le réchauffement de l'hypolimnion. De plus, le pompage étant très irrégulier, l'hypolimnion s'est moins réchauffé : sa température est de $9-10^{\circ} \mathrm{C}$ en juillet. Malgré un épilimnion froid $\left(20^{\circ} \mathrm{C}\right)$ la stratification est bien marquée, ce qui permet une action importante du cisaillement dans la déstratification dès mi-août. Cette phase est accélérée par une reprise de la gestion hydraulique de mi-août à mi-septembre.

\section{V.3.7 Année 1981}

En 1981, l'hiver est froid et le printemps très pluvieux correspond à un pompage régulier, ce qui empêche une thermocline bien marquée de s'établir. Le refroidissement du mois de
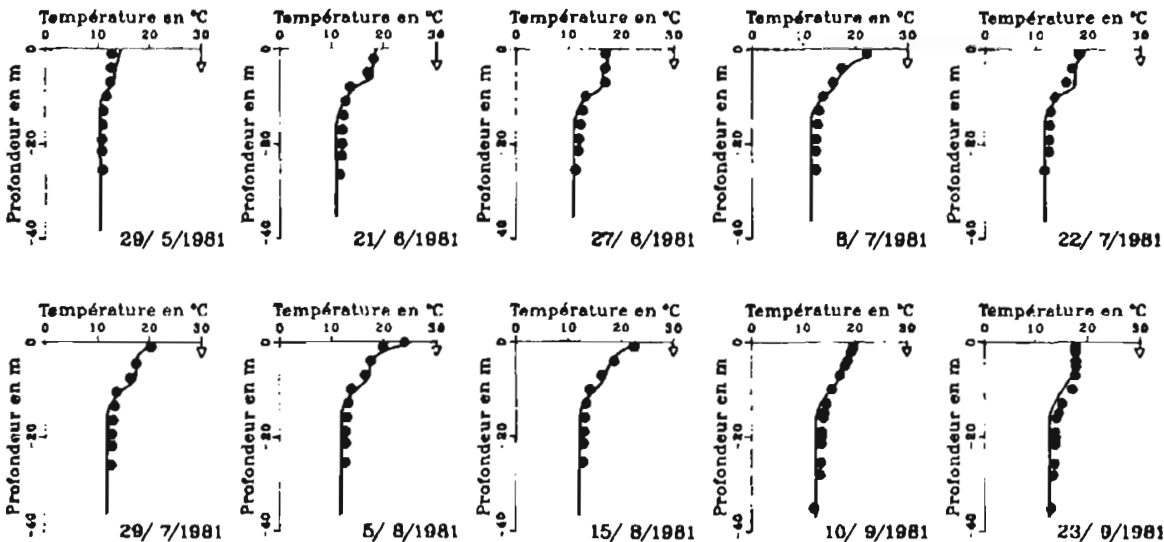

Fig. 15. - Effet d'une prolifération algale intense sur l'absorption de la lumière et sur le profil thermique de Pareloup en 1981. Chaque point correspond aux mesures; le trait continu correspond aux résultats de la simulation; le triangle $(\nabla)$ indique la profondeur du disque de Secchi.

Fig. 15. - Effect of intense algal proliferation on light absorption and on the thermal profile of Pareloup in 1981. Each point corresponds to measurements; the solid line corresponds to simulation results, the Iriangle $(\nabla)$ indicates the Secchi disk depth. 
mai remélange le lac et la stratification ne s'établit que vers fin juin. Cependant, un fort refroidissement en juillet provoque un approfondissement inhabituel, responsable, cette année-là, d'une stabilisation très tardive de la thermocline. L'été est marqué cette année-là par une eutrophisation importante du lac, avec un développement algal intense qui limite la pénétration de la lumière aux tout premiers mètres de surface (fig. 15). L'absence de pompage et turbinage à l'automne, maintient la stratification plus tardivement.

\section{V.3.8 Année 1982}

En 1982, quelques thermoclines transitoires apparaissent en avril, pendant les périodes où le fonctionnement de l'usine est ralenti. La thermocline est en place dès la fin du mois de mai, ce qui est en partie dû à l'arrêt de la gestion de mai à mi-septembre. De ce fait, I'hypolimnion reste froid $\left(9^{\circ} \mathrm{C}\right)$. Le mois de juillet est chaud et peu venté, ce qui favorise un épilimnion très chaud $\left(25^{\circ} \mathrm{C}\right)$ mais très mince $(4 \mathrm{~m})$ (fig. 14). Le refroidissement du mois d'août permet de revenir à des conditions de stratification plus habituelles. En septembre, la reprise de la gestion accélère le mélange.

\section{V.3.9 Année 1983}

La gestion est très active jusqu'à début juin et les refroidissements alternent avec les réchauffements météorologiques. Aussi, la stratification s'amorce fin avril mais le lac se remélange fin mai, ce qui réchauffe l'hypolimnion à $11^{\circ} \mathrm{C}$. La thermocline se stabilise dès mi-juin, alors que la gestion est à l'arrêt jusqu'à fin août et se renforce en juillet, qui est très chaud, avec une température de $25^{\circ} \mathrm{C}$ dans l'épilimnion (fig. 16). La déstratification est mieux reproduite cette année-là, alors que la gestion est à l'arrêt.

\section{V.3.10 Année 1984}

En 1984, le printemps connaît, en avril, une période chaude qui coïncide avec un arrêt prolongé de pompage. La concomitance de ces deux événements favorise la permanence d'une thermocline transitoire relativement profonde (située vers $-11 \mathrm{~m}$ ) pendant une période longue (1 mois environ). Cette année-là, le modèle a tendance à trop stratifier la retenue, ce qui se traduit, à l'automne, par un retard plus marqué dans la déstratification.

\section{V.3.11 Année 1985}

Après un mois de janvier très froid pendant lequel la retenue a pris en glace (fig. 19), le printemps a été frais et pluvieux avec un pompage continu jusqu'à fin mai. La stratification apparaît fin mai mais se stabilise vraiment en juillet (fig. 16). Malgré un été chaud et $\mathrm{sec}$, la température de l'épilimnion se maintient aux environs de $20-21^{\circ} \mathrm{C}$. A l'automne, la déstratification est très bien reproduite, cependant que le pompage est arrêté.

\section{V.3.12 Année 1986}

Après un mois d'avril très froid et un printemps très pluvieux associé à un pompage continu, la stratification dé- 

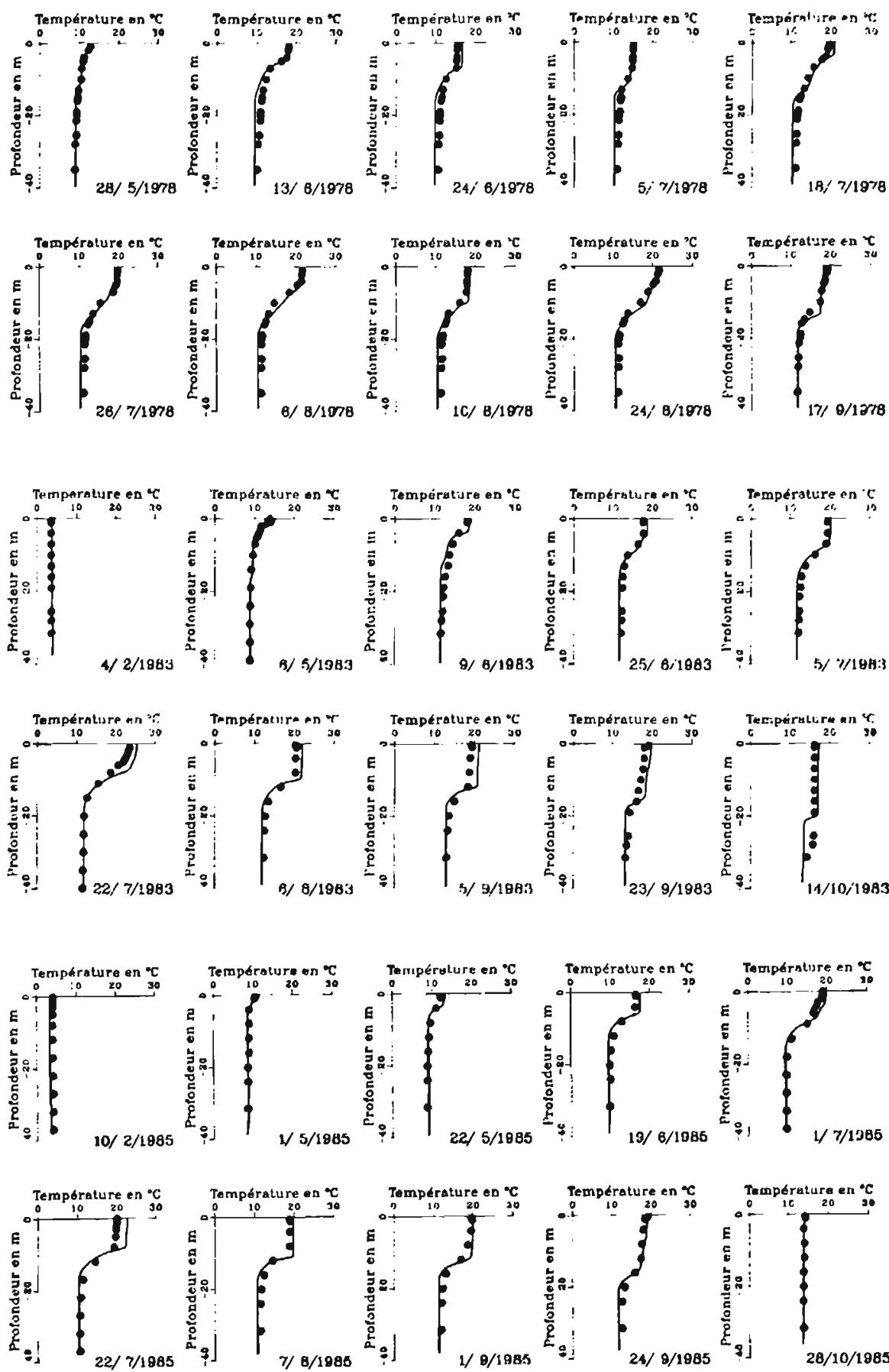

24/ $8 / 1985$

$28 / 10 / 1985$ 

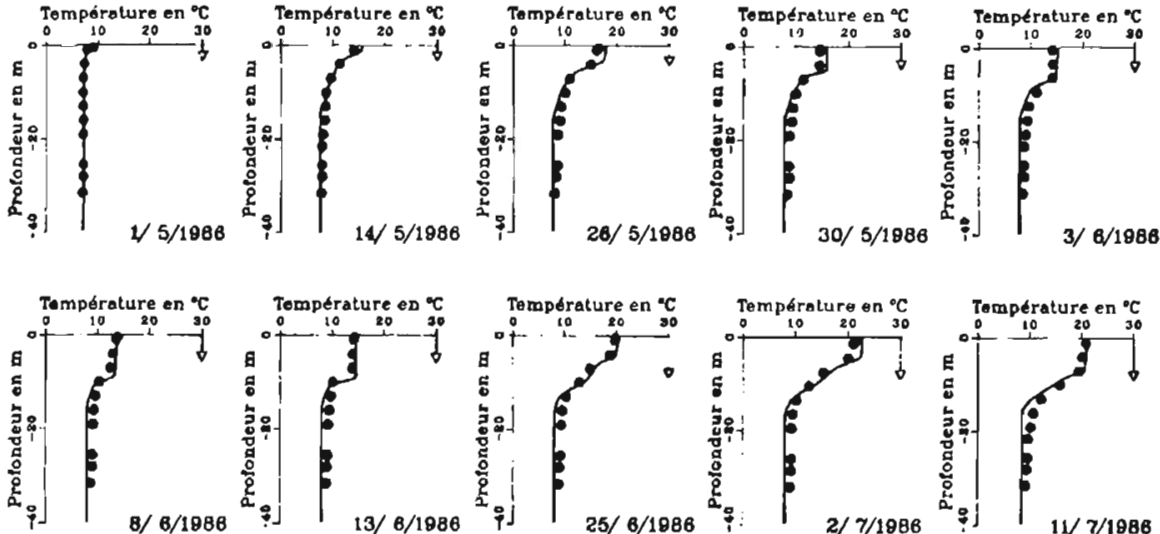

Fig. 17. - Effet d'une variation rapide de la profondeur du disque de Secchi sur le profil thermique de Pareloup en 1986. Chaque point correspond aux mesures; le trait continu correspond aux résultats de la simulation; le triangle $(\nabla)$ indique la profondeur du disque de Secchi.

Fig. 17. - Effect of rapid variation in Secchi disk depth on the thermal profile of Pareloup in 1986. Each point corresponds to measurements; the solid line corresponds to simulation results, the triangle $(\nabla)$ indicates the Secchi disk depth.

marre seulement en mai mais se maintient tout l'été. L'hypolimnion est donc plus froid cette année-là ( 9 à $10^{\circ} \mathrm{C}$ ) et la thermocline bien établie. Juillet et surtout août étant chauds, la température de l'épilimnion est élevée, 24 à $25^{\circ} \mathrm{C}$ en août (fig. 17). La structure thermique de 1986 est proche de celle de 1976, ce qui est essentiellement dû à l'arrêt du pompage de juin à août. Elle est également bien simulée, ce qui montre l'influence considérable du pompage de Bage et de sa température d'entrée.

\section{V.3.13 Année 1987}

Après un mois de janvier très froid pendant lequel la retenue gèle (fig. 19), un début de stratification apparaît en avril mais le printemps étant frais, le lac est remélangé en mai. La stratification s'installe fin mai, alors que la gestion est arrêtée pour travaux de mai à août. La thermocline est bien marquée, comme en 1986 et

Fig. 16. - Retenue de Pareloup: comparaison des profils thermiques calculés (trait continu) et mesurés $(\bullet)$ en 1978,1983 et 1985 . Le réservoir est moins stratifié, le printemps froid et pluvieux et l'été chaud.

Fig. 16. - Pareloup reservoir: comparison of calculated (solid line) and measured ( $(0)$ thermal profiles in 1978, 1983 and 1985. The reservoir is less stratified, the spring cold and rainy, and the summer hot. 
la déstratification bien représentée (fig. 18).

\section{V.3.14 Conclusion}

Le modèle thermique EOLE a été appliqué à la retenue de Pareloup pendant une très longue période : 1976-1987. Le bon accord entre les profils simulés et mesurés sur toute cette période montre que le modèle permet de bien reproduire le régime thermique de cette retenue, en réponse à une grande variabilité de situations.

Lorsque les hivers sont très froids, comme c'est le cas en 1985 et 1987 , une stratification thermique inverse s'installe (fig. 19). Le modèle repré-
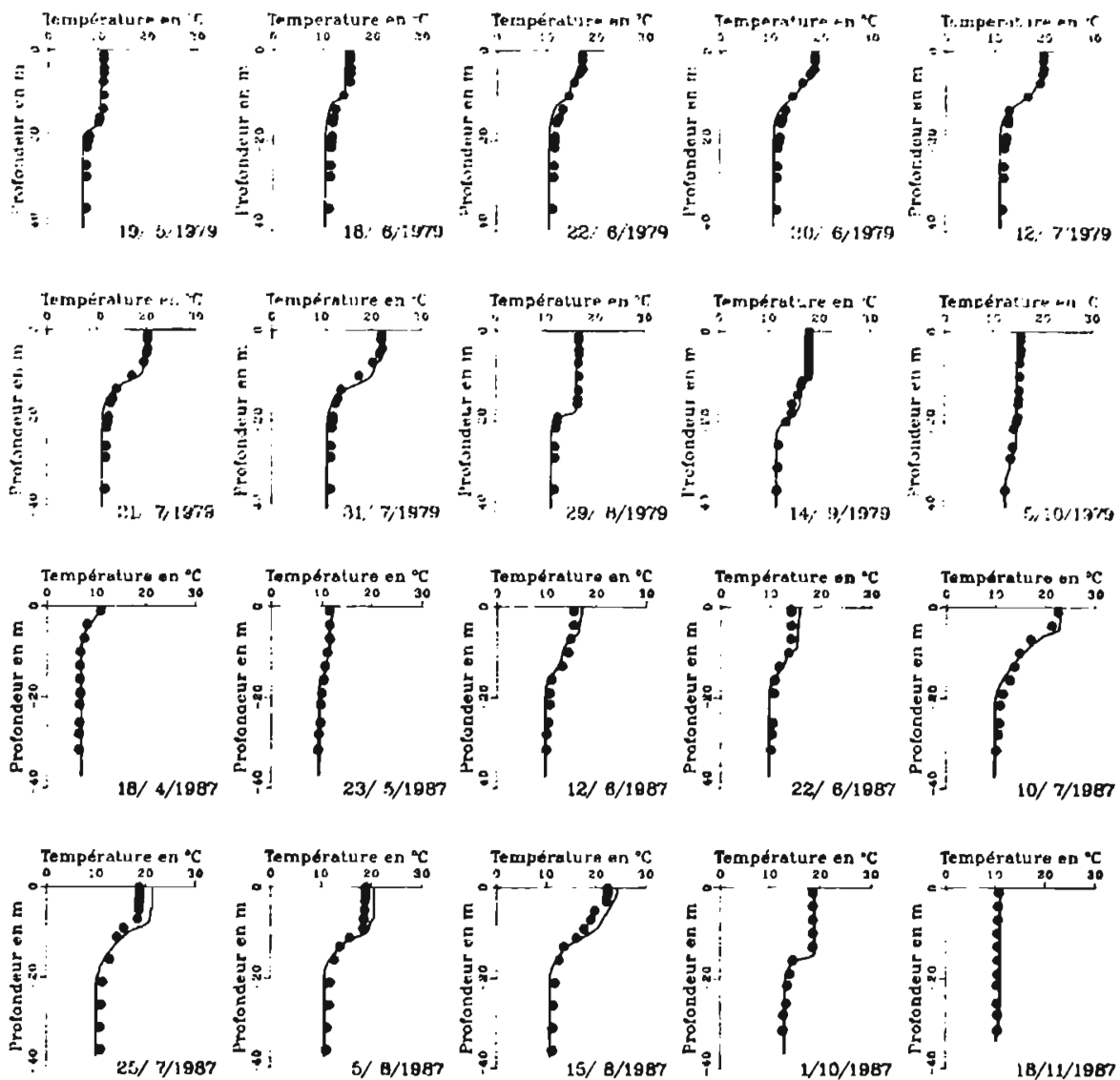

Fig. 18. - Retenue de Pareloup: comparaison des profils thermiques calculés (trait continu) et mesurés (•) en 1979 et 1987 . Le réservoir est bien stratifié, le printemps sec et pas de gestion l'été.

Fig. 18. - Pareloup reservoir: comparison of calculated (solid line) and measured ( $\bullet$ ) thermal profiles in 1979 and 1987. The reservoir is well stratified, the spring dry, and there is no summer turbining. 

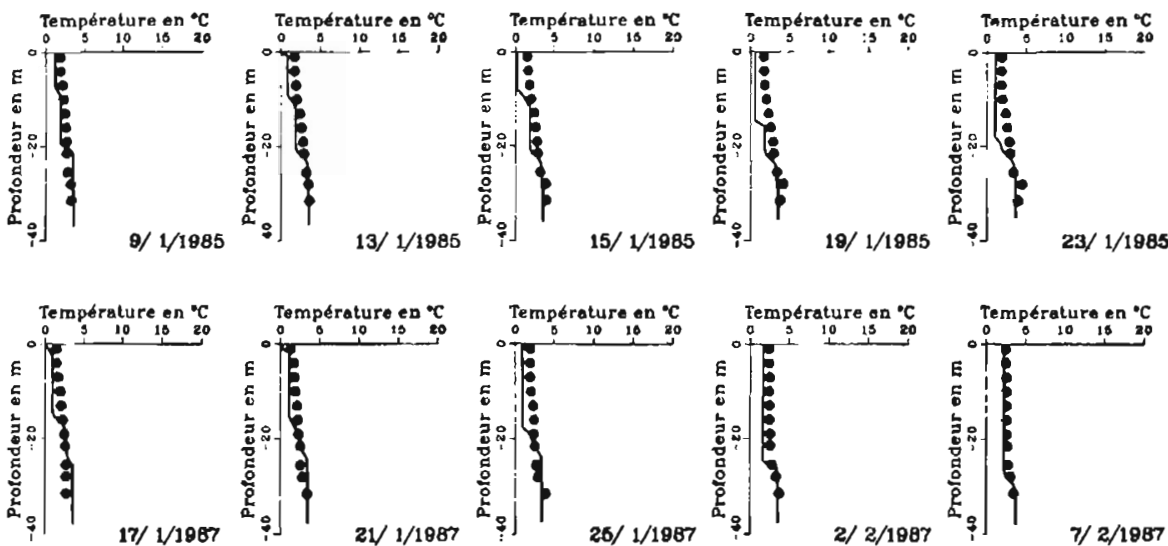

Fig. 19. - Retenue de Pareloup : stratification thermique inverse pendant les hivers 1985 et 1987. Chaque point correspond aux mesures; le trait continu correspond aux résultats de la simulation. Fig. 19. - Pareloup reservoir: inverse thermal stratification during the winters of 1985 and 1987. Each point corresponds to measurements; the solid line corresponds to simulation results.

sente bien ce refroidissement de surface tant que la température n'atteint pas $0^{\circ} \mathrm{C}$. A partir de ce moment-là, une couche de glace se développe en surface et modifie les échanges atmosphériques. Le modèle n'est pas conçu pour simuler cette prise en glace et continue à refroidir la masse d'eau, prédisant alors une température de surface trop froide. Cependant, cette légère divergence est rattrapée dès le réchauffement climatique. Comme ces épisodes restent exceptionnels et de courte durée, nous n'avons pas modifié l'algorithme de refroidissement.

L'analyse des résultats du modèle nous permet de remarquer l'influence conjuguée des conditions météorologiques et hydrologiques printanières sur la dynamique de la stratification. En effet, au printemps, l'apport d'ECT par le vent est dominant et détermine, avec la convection pénétrante, l'installation de la stratification. La gestion hydraulique joue alors un rôle important: un arrêt momentané du pompage contribue fortement à la stabilisation de la thermocline, d'une part en ne réchauffant pas I'hypolimnion, d'autre part en n'apportant pas de turbulence, alors qu'une augmentation des débits turbinés, pendant une période froide ou humide, par exemple, favorise le mélange vertical. Or, en général, la gestion hydraulique est très étroitement associèe aux conditions hydrométéorologiques: une période chaude et sèche correspond à une consommation énergétique moindre, donc à de faibles débits, alors qu'inversement, un printemps froid et humide est associé à de forts débits. Le modèle reproduit très bien cette phase printanière dans tous les cas rencontrés. 
Lorsque le printemps comporte des périodes chaudes et non ventées relativement longues, de l'ordre d'une semaine, la phase de stratification transitoire est prolongée, isolant momentanément l'hypolimnion. En 1980, de telles situations se renouvellent souvent au cours du printemps, ralentissant le réchauffement de l'hypolimnion. En effet, c'est surtout l'alternance rapide des phases de stratification et de mélange qui permet de réchauffer progressivement l'hypolimnion tout au long du printemps. Simultanément, le pompage très irrégulier ne permet pas à la température de I'hypolimnion de dépasser $9^{\circ} \mathrm{C}$. En 1982 et 1984, c'est la concomitance, en avril, d'une période chaude et d'un arrêt prolongé du pompage qui stabilise très tôt la thermocline transitoire, ce qui favorise une stratification marquée en été, caractérisée par un hypolimnion froid. Les années 1976, 1986 et 1987, bien que particulières, correspondent à des situations voisines: la gestion hydraulique a été complètement arrêtée de mai à août, en raison d'une forte sécheresse en 1976, et de travaux effectués sur les conduites en 1986 et 1987. Au cours de ces trois années, le comportement de Pareloup a été proche de celui d'un lac naturel, avec un épilimnion chaud $\left(24^{\circ} \mathrm{C}\right)$, une thermocline bien marquée et un hypolimnion froid $\left(8\right.$ à $\left.9^{\circ} \mathrm{C}\right)$. Ces années-là, la mise en place de la stratification est particulièrement bien reproduite, confirmant que les mécanismes de mélange reliés aux échanges de surface sont bien pris en compte dans le modèle.
Lorsque le printemps est froid et pluvieux, le pompage et le turbinage sont généralement intenses et réguliers: c'est le cas en 1977, 1981, 1983 et 1985. La stratification s'établit tardivement, après une succession de périodes de mélange complet du lac. L'hypolimnion est alors plus chaud en été, de l'ordre de 11 à $12^{\circ} \mathrm{C}$.

La thermocline saisonnière, généralement installée entre le 1 et le 15 juillet, reste stable tout l'été, ce que favorise une gestion hydraulique réduite et souvent même arrêtée, afin de maintenir le plan d'eau à niveau constant pendant la période touristique. Aussi, la température de l'hypolimnion évolue très peu en été. Sa bonne représentation montre la bonne simulation de la dynamique de la thermocline mais également celle de l'advection des masses d'eau, en particulier celles du pompage de Bage qui alimentent directement les couches profondes.

Si l'hypolimnion est déterminé par les conditions printanières, les caractéristiques de la couche de surface dépendent du climat estival. En raison de l'altitude, les étés sont frais à $\mathrm{Pa}$ reloup et la température de la couche de surface se situe au voisinage de 20 à $21^{\circ} \mathrm{C}$ et dépasse rarement $24^{\circ} \mathrm{C}$. Là encore, les résultats du modèle sont en bon accord avec les mesures. En 1976, 1982 et 1986, le mois de juillet a été très chaud et la température de surface a atteint $25^{\circ} \mathrm{C}$. En $1977,1979,1980$ et 1984 , la température de l'eau de surface n'a pas dépassé $20^{\circ} \mathrm{C}$ car l'été a été frais. En 
1981, un fort refroidissement en juillet a provoqué un approfondissement inhabituel, responsable, cette année-là, d'une stabilisation très tardive de la thermocline. De plus, l'été a été marqué par une eutrophisation importante du lac, avec un développement algal intense qui a limité la pénétration de la lumière aux tous premiers mètres de surface.

Le mélange automnal démarre vers mi-septembre et a lieu très rapidement, car le refroidissement climatique d'automne est très brusque à Pareloup, associé à de violents coups de vent du Nord. De plus, l'automne correspond souvent à la reprise d'une gestion hydraulique plus intense qui accélère cette phase de mélange. Si le début de la déstratification est bien reproduit dans la simulation, on observe alors un léger retard dans la dynamique du mélange. Ce retard, systématiquement constaté sur toutes les années de la période d'étude, signale qu'un processus important à ce moment-là n'est pas correctement représenté, ce qui se traduit par un déficit d'énergie cinétique turbulente disponible pour le mélange.

Dans le modèle, la déstratification est due aux apport d'ECT en surface : la convection pénétrante qui devient dominante à cette époque de fort refroidissement atmosphérique, associée au cisaillement du vent. II semble donc que les seuls processus de surface ainsi représentés ne puissent, à eux seuls, simuler correctement la déstratification.

Nous avons observé, lors des traçages réalisés à l'automne (Salençon et al., 1988; Salençon et Calmels, 1994), que le cisaillement à la thermocline créé par les circulations induites par le vent est important. Sa participation active à l'érosion du gradient thermique semble en faire un des principaux facteurs de déstratification à Pareloup. Cette source de turbulence est assez délicate à introduire dans un modèle saisonnier (Spigel et al., 1986) et n'est pas prise en compte explicitement dans le modèle actuel. Sa représentation très schématique (une compensation immédiate de la dissipation) la relie directement aux apports de surface et ne tient pas compte de l'effet tampon joué implicitement par les circulations baroclines, qui, une fois établies, permettent de soutenir l'érosion du gradient thermique après l'arrêt du coup de vent. D'autre part, nous n'avons pas non plus introduit de diffusion hypolimnique reliee aux apports d'ECT de surface, ce qui permettrait d'éroder le gradient thermique et de rendre plus efficace l'ECT disponible.

Nous n'avons pas modélisé pour l'instant ces deux processus turbulents afin de ne pas alourdir le modèle, dont l'objectif est le couplage avec un modèle biologique. Cependant, certaines espèces phytoplanctoniques se développant au niveau de la thermocline, vers la fin de l'été, sont très sensibles à la dynamique de la thermocline dont dépend également l'ampleur de la croissance algale automnale (Salençon et Thébault, 1995). Aussi envisageons-nous d'introduire ces deux processus turbulents dans 
le modèle thermique, pour une meilleure représentation de l'écosystème.

Cependant, malgré cette faiblesse ponctuelle, le comportement global du modèle sur une aussi longue période reste très satisfaisant.

Le bon accord entre les températures de sortie, calculées et mesurées, pour le turbinage d'Alrance comme pour le débit réservé du Vioulou, confirme la bonne représentation du modèle thermique sur une aussi longue période (fig. 20).

\section{V.4 Discussion}

Nous devons insister sur l'importance de la qualité des données d'entrée utilisées pour les simulations. En effet, l'ECT apportée par le vent dépend directement de la vitesse du vent. Une série de mesures de vent non représentatives des conditions locales peut induire une structure thermique erronée qui restera inscrite pour le reste de l'année et pourra perturber la mise en place correcte de la thermocline saisonnière. Dans un système "à mémoire" comme les lacs et réservoirs, il est donc particulièrement important de disposer de données météorologiques et hydrologiques de bonne qualité et représentant bien les conditions locales.

II faut également noter l'influence du pas de temps d'acquisition des données météorologiques. En effet, comme le remarque Imberger (1979), la prise en compte d'un vent moyen représente une perte d'énergie considérable : par exemple, un vent moyen journalier constant de $5 \mathrm{~m} . \mathrm{s}^{-1}$ apporte 16 fois moins d'énergie qu'un vent de $20 \mathrm{~m} \cdot \mathrm{s}^{-1}$ soufflant pendant 6 heures, même si les moyennes journalières sont identiques. II est donc très important de disposer de données météorologiques avec un pas de temps au moins trihoraire et, si possible, horaire pour le vent.

Enfin, nous insistons sur la nécessité de disposer de mesures in situ du coefficient d'extinction. Influencé par la biomasse, l'été, et par la mise en suspension des sédiments, l'hiver, il varie de façon considérable au cours du cycle annuel et joue un rôle fondamental sur l'absorption de la lumière et la structure thermique verticale (fig. 17).

Dans ces conditions, pourvu que les données d'entrées soient bien représentatives du site étudié, le modèle n'a pas besoin de calage car il est basé sur la représentation des processus: dans les différents cas d'application, les coefficients du modèle sont identiques (Salençon, 1994).

\section{CONCLUSION}

Le modèle vertical hydrodynamique et thermique EOLE, a été développé pour simuler l'évolution de la stratification thermique d'un réservoir en tenant compte des échanges thermiques à l'interface air-eau ainsi que des entrées et sorties d'eau. L'objectif de ce modele est de servir de base à un modèle d'écosystème, ce qui a 

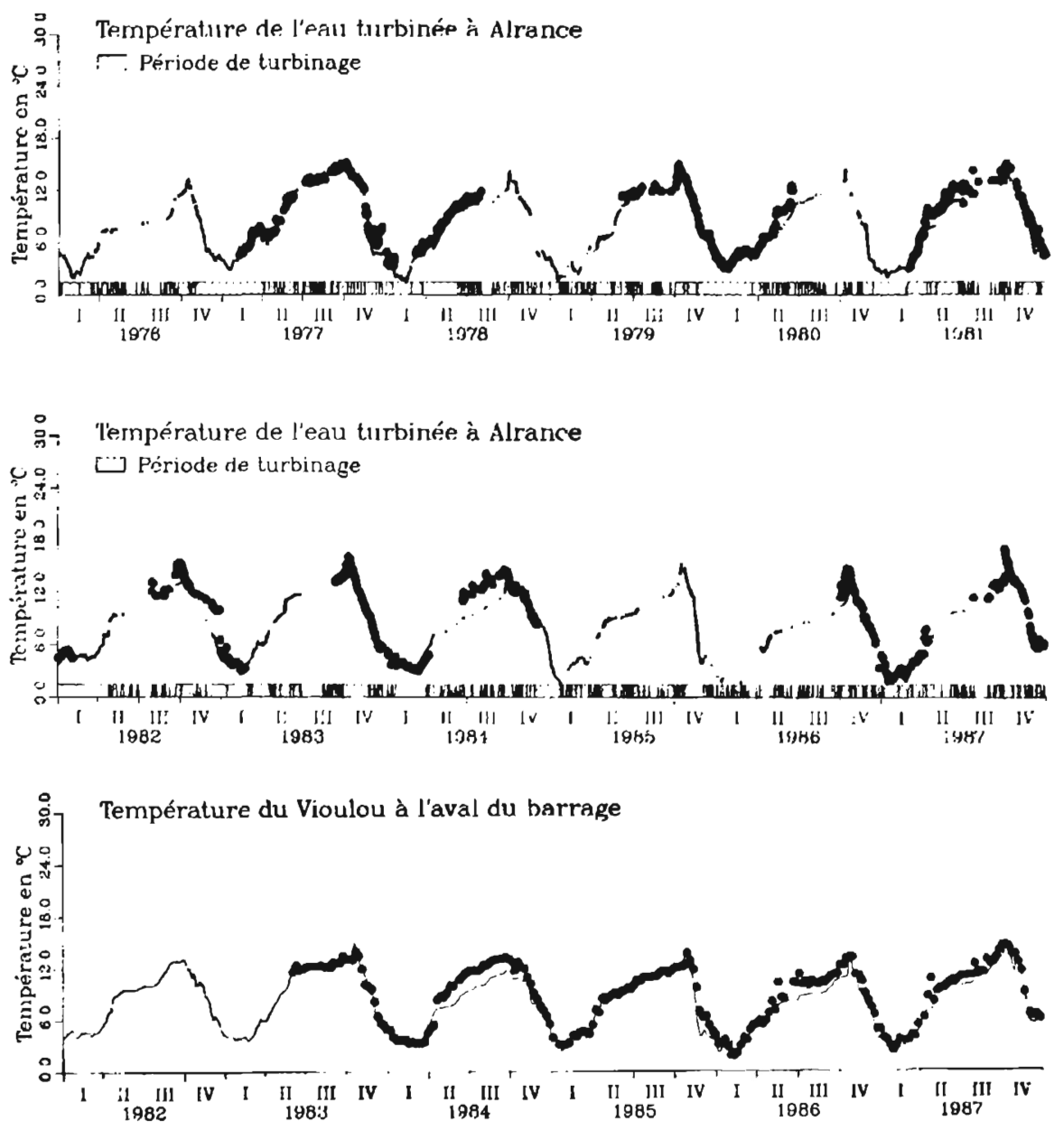

Fig. 20. - Comparaison des températures de sortie calculèes (trait continu) et mesurées $(\bullet)$ : turbinage d'Alrance et débit réservé du Vioulou.

Fig. 20. - Comparison of calculated (solid line) and measured (•) outflow temperatures: water turbined at Alrance and residual stream flow in the Vioulou.

orienté certains choix de modélisation, en particulier, l'unidimensionnalité verticale, le choix d'un modèle intégral à couche de mélange basé sur la représentation des processus, la discrétisation en volumes finis.
Ce modèle a permis de très bien reproduire, en comparaison avec des mesures in situ, la stratification thermique de cette retenue, la dynamique verticale des masses d'eau, le régime thermique des eaux restituées à l'aval, 
ainsi que les fluctuations interannuelles sur de longues périodes (plusieurs cycles annuels). Appliqué ensuite à des réservoirs de caractéristiques très différentes, il a permis, dans chaque cas, d'obtenir de bons résultats, en utilisant les mêmes valeurs des coefficients. La précision des résultats alliée à la relative simplicité de la représentation nous a permis d'utiliser ce modèle comme structure de base d'un modèle d'écosystème.

\section{RÉFÉRENCES \\ BIBLIOGRAPHIQUES}

Agoumi A., Salençon M.J. et Gosse Ph., 1985. Etude de la répartition de la chlorophylle sur la verticale en Manche occidentale: Utilisation d'un modèle multicouche. Rapport HE31/85-35, Electricité de France, Paris, $35 \mathrm{pp}$.

Akiyama J. and Stefan H.G., 1984. Plunging flow into a reservoir: Theory. J. Hydraul. Eng., ASCE, 110(4) : 484-499.

Bagnouls F. et Gaussen H., 1953. Saison sèche et indice xérothermique. Bull. Soc. Hist. nat. Toulouse, 88 : 193-239.

Bosc P., 1985. Evaluation des apports en éléments nutritifs du bassin versant dans le lac de Pareloup. Mémoire de DEA, Ecologie. Université Paul Sabatier, Toulouse, $123 \mathrm{pp}$.

Calmels P. et Salençon M.J., 1991. Techniques de marquage pour l'étude des circulations des masses d'eau dans les lacs. Application à la retenue de Pareloup. In: IAEA (Editor), Use of Isotope Techniques in Water Resources Development. Proc. Symp. 11-15 March 1991, Vienna. IAEA UNESCO, Vienna, pp. 107-122.

Chabard J.P., Daubert O. et Goutal N., 1989. Cours de simulation numérique du LNH. Notions de base sur l'analyse nu- mérique (Tome 1). Rapport HE41/8930, Electricité de France, Paris, $75 \mathrm{pp}$.

Destuynder P., 1986. Modélisation de l'évolution de l'épaisseur d'un couvert de glace sur une retenue de montagne. Rapport HE31/86-01, Electricité de France, Paris, $31 \mathrm{pp}$.

Destuynder-Bélus P., 1989. Evolution thermique du Lac de Valdai: Calage du modèle simulant l'évolution temporelle de l'épaisseur de glace sur une retenue. Rapport HE31/89-07, Electricité de France, Paris, 11 pp.

Enderlé M.J., 1980. Détermination du profil vertical de température d'une retenue de barrage: application à la retenue de Grangent sur la Loire. Rapport HE-31/80.33, Electricité de France, Paris, $16 \mathrm{pp}$.

Enderlé M.J., 1982. Impact of a pumped storage station on temperature and water quality of the two reservoirs. In : S.E. Jrgensen (Editor), Third International Conference on State of the Art in Ecological Modelling, 24-28 May 1982, Fort Collins. ISEM Journal, 4(34) : 87-95.

Enderlè-Salençon M.J., 1983. Etude de l'impact de l'aménagement de la pompe d'Alrance sur le régime thermique du lac de PARELOUP. Rapport HE31/83-11, Electricité de France, Paris, $17 \mathrm{pp}$.

Enderlé M.J. and Gras R.A., 1983. Hydraulic processes influencing phytoplanktonic growth in a reservoir : wind mixing, inflows, outflows. In : V.I.N.I.T.I. (Editor), 20 ${ }^{\text {th }}$ IAHR Congress on Fluid Mechanics in Energy Production. Proc. Symp., 5-9 sept 1983, Moscow. Ail Union Institute for Scientific and Technical Information (V.I.N.I.T.I.), Moscow, $6: 570-586$.

Ford D.E. and Stefan H.G., 1980. Thermal prediction using integral energy model. J. Hydraul. Div. ASCE, $106: 39-55$.

Gaillard J., 1981. A predictive model for water quality in reservoirs and its application to selective withdrawal. $\mathrm{Ph}$. 
D. Dissertation. Colorado State University, Fort Collins, $232 \mathrm{pp}$.

Garçon V., 1981. Modélisation numérique d'un écosystème aquatique : application au réservoir de Grangent sur la Loire. Thèse de $3^{e}$ cycle. Université de Paris VII, Paris, $230 \mathrm{pp}$.

Gilbert A. et Salençon M.J., 1988. Etude themo-haline de la retenue de Mirgenbach à Cattenom. Rapport HE31/88-32, Electricité de France, Paris, 34 pp.

Gilbert A., Gras R.A. and Roult D., 1986. Numerical computation of natural river temperatures. In : BHRA and The Fluid Engineering Centre (Editors), International Conference on Water Quality Modelling in the Inland Natural Environment. Proc. Symp., 10-13 June 1986, Bournemouth. BHRA and The Fluid Engineering Centre, Cranfield, M1 : 457-471.

Harleman D.R.F., 1982. Hydrothermal analysis of lakes and reservoirs. J. Hydraul. Div. ASCE, 108 : 302-325.

Hebbert B., Imberger J., Loh I. and Patterson J.C., 1979. Collie river underflow into the Wellington reservoir. J. Hydraul. Div. ASCE, 105 : 533-545.

Hocking G.C., Sherman B.S. and Patterson J.C., 1988. Algorithm for selective withdrawal from stratified reservoir. J. Hydraul. Div. ASCE, 114(7) : 707-719.

Hurley Octavio K.A., Jirka G.H. and Harleman D.R.F., 1977. Vertical heat transport mechanisms in lakes and reservoirs. M.I.T. Hydrodynamics Laboratory, Boston, Technical Report $\mathrm{N}^{\circ} 227,126 \mathrm{pp}$.

Imberger J., 1979. Mixing in reservoirs. In : H.G. Fischer, E.J. List, R.C.Y. Koh, J. Imberger and N.H. Brooks (Editors), Mixing in Inland and Coastal Waters. Academic Press, New York, pp. 148-228.

Imberger J. and Patterson J.C., 1989. Physical Limnoly. In: T. Wu (Editor), Advances in applied mechanics. Academic Press, New York, pp. 303-475.

Imberger J., Patterson J., Hebbert B. and Loh I., 1978. Dynamics of reservoir of medium size. J. Hydraul. Div. ASCE, $104: 725-743$.

Klein J.P. et Bonnaud E. 1977. Détermination du profil vertical de température d'une masse d'eau à partir de données météorologiques. Rapport HE31/77-19, Electricité de France, Paris, $22 \mathrm{pp}$.

Markofsky M. and Harleman D.R.F., 1971. A predictive model for thermal stratification and water quality in reservoirs. M.I.T. Hydrodynamics Laboratory, Boston, Technical Report No 134, 283 pp.

Niiler P.P. and Kraus E.B., 1977. One-dimensionnal models of the upper ocean. In : E.B. Kraus (Editor), Modelling and Prediction of the upper layers in ocean. Pergamon Press, New York, pp. 143-172.

Patterson J.C., Hamblin P.F. and Imberger J., 1984. Classification and dynamic simulation of the vertical density structure of lakes. Limnol. Oceanogr., 29 : 845-861.

Pourcher A.M. et Salençon M.J., 1990. Modélisation du plancton dans une retenue oligotrophe: Sainte-Croix sur le Verdon. Hydroécol. Appl., 2(1/2) : 91-134.

Pujo H., 1994. Levé bathymétrique du réservoir de Pareloup. Hydroécol. Appl. 6(1/2) : 9-17.

Rayner K.N., 1980. Diurnal energetics of a reservoir surface layer. University of Western Australia, Environ. Dynam., Nedlands, Technical Report n ED-80005, 227 pp.

Ryan P.J. and Harleman D.R.F., 1971. Prediction of the annual cycle of temperature changes in a stratified lake or reservoir: mathematical model and user's manual. M.I.T. Hydrodynamics Laboratory, Boston, Technical Report $\mathrm{n}^{\circ} 137,132 \mathrm{pp}$.

Salençon M.J., 1986. Modélisation de l'eutrophisation des lacs et retenues. In: Symposium sur la Qualité des Eaux de Surface EHTP. Proc. Symp., 20-21 Nov 1986, Casablanca. Eau et Développement, Casablanca, $4: 68-88$ et 
Rapport HE31/86-8, Electricité de France, Paris, $31 \mathrm{pp}$.

Salençon M.J., 1992. Etude de la qualité de l'eau des retenues de Rochebut et Chambonchard - Synthèse des résultats. Rapport HE31/92-7, Electricité de France, Paris, $20 \mathrm{pp}$.

Salençon M.J., 1994. Stratification thermique d'un réservoir : le modèle à bilan d'énergie, EOLE. Rappont HE31/94-1, Electricité de France, Paris, $113 \mathrm{pp}$.

Salençon M.J. et Calmels P., 1994. Etude de la dynamique des masses d'eau du lac de Pareloup par traçages. Hydroécol. Appl. 6(1/2) : 19-58.

Salençon M.J. et Destuynder-Bélus P., 1987. Modèles de qualité d'eau des réservoirs: exemples de réponses concrètes. In : W.H. Graf and U. Lemmin (Editors), 22 ${ }^{\text {th }}$ IAHR Congress on Topics in Lake and Reservoir Hydraulics. Proc. Symp., 31 August-4 Sept 1987, Lausanne. Ecole Polytechnique Fédérale, Lausanne, pp. 187-193.

Salençon M.J. et Gilbert A., 1991. Etude de la qualité de l'eau des retenues de Rochebut et Chambonchard: Modélisation thermique. Rapport HE31/9128. Electricité de France, Paris, $50 \mathrm{pp}$.

Salençon M.J. et Simonot J.Y., 1989. Proposition de modèle bidimensionnel ( $x$ $z$ ) dynamique et thermique de retenue. Rapport HE31/89-21, Electricité de France, Paris, $34 \mathrm{pp}$.

Salençon M.J. et Thèbault J.M., 1994a. Modélisation de l'écosystème du Lac de Pareloup (Aveyron), In : R. Pourriot et M. Meybeck (Editors), Limnologie Générale, Masson, Paris, (sous presse).

Salençon M.J. et Thébault J.M., 1994b. Démarche de modélisation d'un écosysteme lacustre: application au Lac de Pareloup. Hydroécol. Appl. 6(1/2) : 315-327.

Salençon M.J. et Thébault J.M., 1994c. Modélisation de l'écosystème du Lac de Pareloup avec les modèles ASTER et MELODIA. Hydroécol. Appl. 6(1/2) : 369-426.
Salençon M.J. and Thébault J.M., 1995. Simulation model of a mesotrophic reservoir (Lac de Pareloup) : MELODIA, an ecosystem management reservoir model. Ecol. Modelling, (sous presse).

Salençon M.J., Pujo H., Calmels P. et Marion D., 1988. Traçage des masses d'eau de la retenue de Pareloup : septembre 1987. Rapport HE31/88-15, Electricité de France. Paris, $28 \mathrm{pp}$.

Salençon M.J., Thébault J.M. et Capblancq J., 1990. Etude de la retenue de Pareloup Synthèse des travaux réalisés dans le cadre de la Convention EDF-Ministère de l'Environnement (mars 1986-mars 1990), Rapport HE-31/90.23, Electricité de France, Paris, $40 \mathrm{pp}$.

Simons T.J.,1980. Circulation models of lakes and inland seas. Can. Bull. Fish. Aquat. Sci. 230 : 146 pp.

Spigel R.H. and Imberger J., 1980. The classification of mixed-layer dynamics in lakes of small to medium size. J. Phys. Oceanogr., 10: 1104-1121.

Spigel R.H., Imberger J. and Rayner K.N., 1986. Modeling the diurnal mixed layer. Limnol. Oceanogr., $31: 533-556$.

Stefan H.G. and Ford D.E., 1975. Temperature dynamics in dimictic lakes. J. Hydraul. Div. ASCE, 101: 97-114.

Tassin B., 1986. Contribution à la modélisation du lac Léman. Thèse de Docteur Ingénieur. CERGRENE - Ecole Nationale des Ponts et Chaussées, Paris, $189 \mathrm{pp}$.

Vinçon-Leite B., Mouchel J.M. et Tassin B., 1989. Modélisation de l'évolution thermique saisonniere du lac du Bourget. (Savoie, France). Rev. Sci. Eau, 2(4) : 483-510.

Yanenko N.N., 1968. Méthode à pas fractionnaires - Résolution de problèmes polydimensionnels de physique mathématique. Armand Colin, Paris, $205 \mathrm{pp}$. 